Canadian University Music Review

Canadian University Music Review

Revue de musique des universités canadiennes

\title{
Essai de Typologie des Marches d'Harmonie dans la Musique Tonale de Bach à Wagner
}

\section{Luce Beaudet}

Volume 9, numéro 1, 1988

URI : https://id.erudit.org/iderudit/1014923ar

DOI : https://doi.org/10.7202/1014923ar

Aller au sommaire du numéro

Éditeur(s)

Canadian University Music Society / Société de musique des universités canadiennes

ISSN

0710-0353 (imprimé)

2291-2436 (numérique)

Découvrir la revue

Citer cet article

Beaudet, L. (1988). Essai de Typologie des Marches d'Harmonie dans la Musique Tonale de Bach à Wagner. Canadian University Music Review / Revue de musique des universités canadiennes, 9(1), 19-82.

https://doi.org/10.7202/1014923ar

All Rights Reserved (C Canadian University Music Society / Société de musique des universités canadiennes, 1988
Ce document est protégé par la loi sur le droit d'auteur. L’utilisation des services d'Érudit (y compris la reproduction) est assujettie à sa politique d'utilisation que vous pouvez consulter en ligne.

https://apropos.erudit.org/fr/usagers/politique-dutilisation/ 


\section{ESSAI DE TYPOLOGIE}

DES MARCHES D'HARMONIE

DANS LA MUSIQUE TONALE

DE BACH À WAGNER

\section{Luce Beaudet}

Une manie de ranger.

De façon à pouvoir repérer.

À l'oreille.

À l'oeil.

Pour que l'oreille devienne oeil et que l'oeil devienne oreille.

Rien ne saurait mieux se prêter à cette fin que la marche d'harmonie. Une pure mécanique de symétrie, faite pour être entendue comme telle et qu'on retrouve à toutes les deux pages du discours tonal, de Bach à Wagner. Aussi semble-t-il étonnant que jusqu'ici, à notre connaissance, aucune démarche de classification de cet objet n'ait été tentée, du moins publiée. Classer donc, mais aussi scruter cet objet comme porteur privilégié d'habitudes et de comportements tonaux, voilà notre propos.

Qu'est-ce que la marche d'harmonie tonale? Sera considérée comme telle toute reproduction (R) systématique immédiate, à un palier supérieur ou inférieur de l'échelle sonore (tonale), d'un modèle (M) correspondant à un ensemble harmonique d'au moins 2 termes (fonctions) : 1

1 Le type de marche auquel se rattachent les exemples musicaux est chaque fois indiqué s'il y a lieu, et dès maintenant, pour permettre au lecteur d'y revenir lorsqu'il aura pris connaissance de la procédure de classification proposée. 


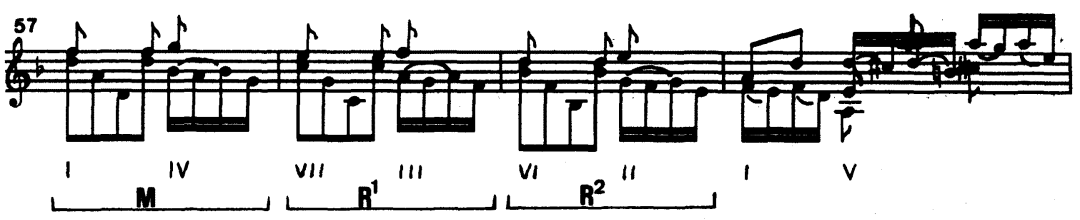

Figure 1. : J. S. Bach : Partita no 2 pour violon seul, "Chaconne", mes. 57-59. Marche de type no 1.
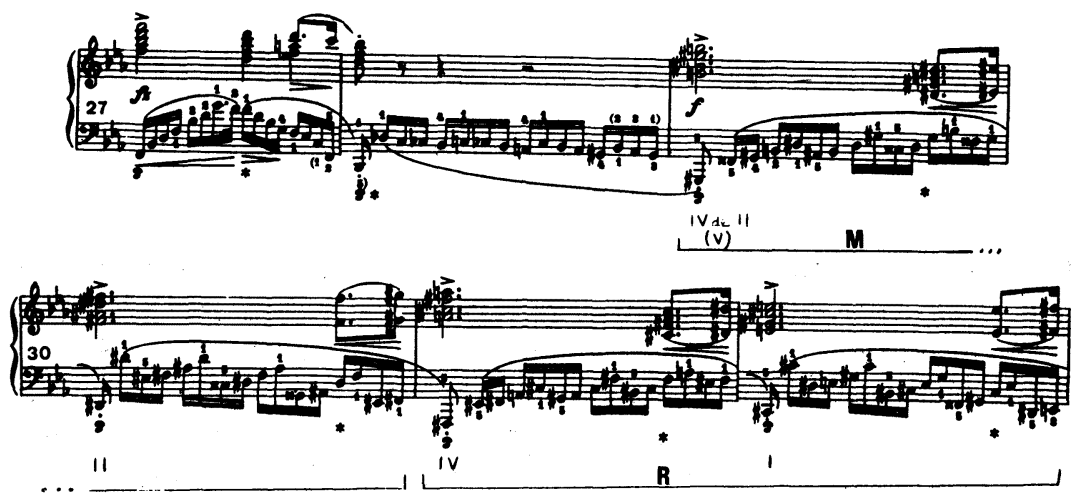

Figure 2. : Chopin : Étude op. 10, no 12, mes. 29-32. Marche de type no 3. 

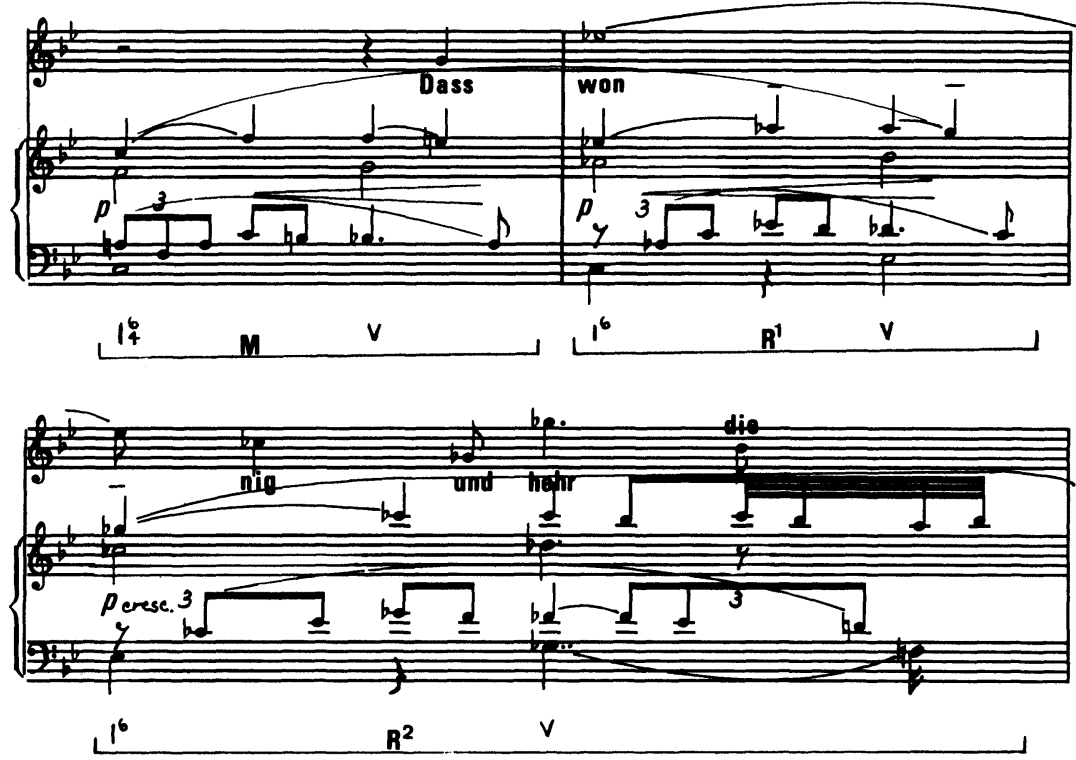

Figure 3. : Wagner : Tristan und Isolde, acte III, scène 2. Marche de type no 10.

Cette définition commande plusieurs remarques. D'abord, on exclut la marche dans laquelle le modèle renvoie à un seul accord, même si les configurations mélodiques et rythmiques sont reproduites symétriquement. En un tel cas, on peut considérer qu'il n'y a pas de modèle harmonique proprement dit et qu'il s'agit là plutôt d'une marche mélodique. ${ }^{2}$

2 En effet, dans la perspective du discours tonal, défini comme discours hiérarchisé, une entité harmonique doit, pour exister, pour être signifiante, désigner la tonique à laquelle elle se rapporte et comporte, à cette fin, au moins deux fonctions (explicites ou implicites) dont la dominante. Un accord isolé, même s'il désigne une structure nettement différenciée - comme celle, par exemple, d'un accord parfait majeur - représente une entité non signifiante à cet égard. 
Cette situation se présente le plus souvent à l'occasion de l'emploi d'une formule harmonique ${ }^{3}$ - par exemple, I-VI-IV-II-V-I - où le traitement du premier accord est repris pour chacun des accords suivants :

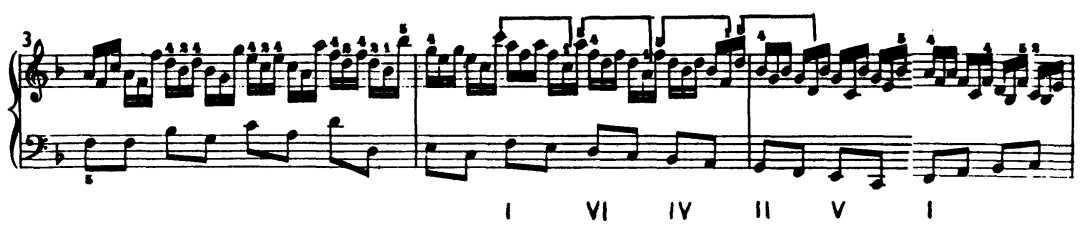

Figure 4. : J. S. Bach : Clavier bien tempéré, vol. 1, "Prélude no 6", mes. 4-5.

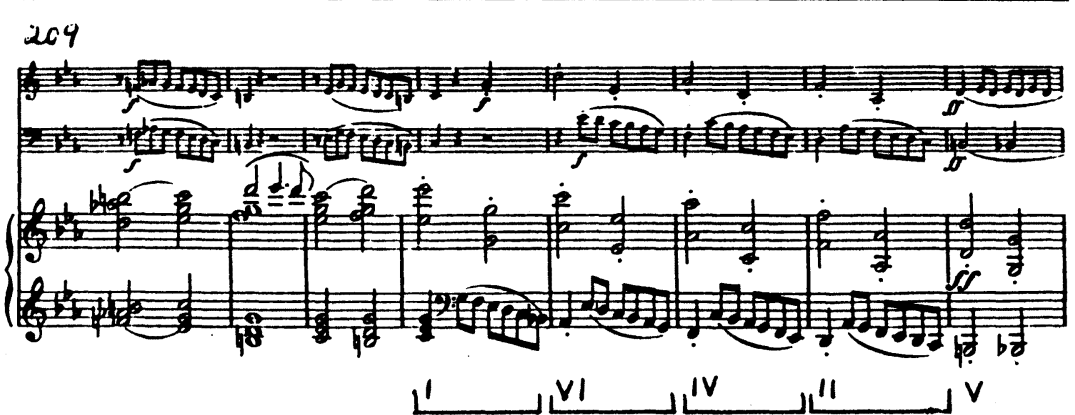

Figure 5. : Beethoven : Trio op. 1, no 3, IV, mes. 212-216.

3 La formule harmonique, telle que nous l'entendons ici, correspond à un fragment de discours harmonique à fort taux de récurrence dans le répertoire tonal et constitué d'au moins trois fonctions (explicites ou implicites) dont la signification se ramène à la désignation sans équivoque d'une tonique spécifique principale ou secondaire. 
On retrouve également le même cas d'une marche à un accord dans le modèle au moment d'un parallélisme d'accords de sixte (ou d'accords de quinte) :

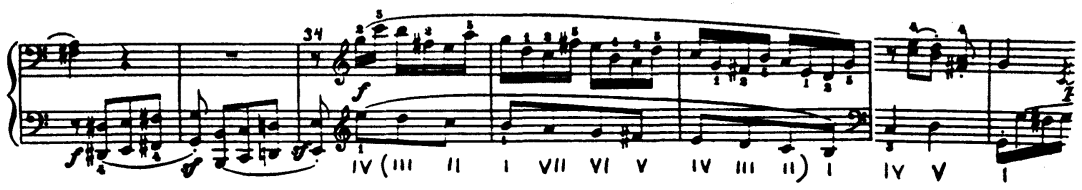

Figure 6. : Mozart : Sonate no 8 (K. 189d), III, mes. 34-36.

On accepte, à l'inverse, de considérer comme marche d'harmonie un fragment de discours où le modèle harmonique seul est répété (transposé) alors que les configurations rythmiques et mélodiques en sont modifiées. Cette extension va permettre d'expliciter plusieurs gestes harmoniques qui, autrement, résistent souvent à l'analyse :

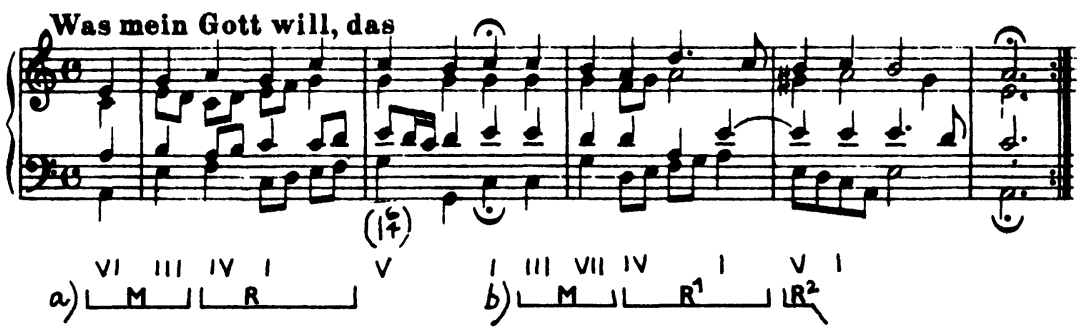

Figure 7. : J. S. Bach: Choral no 41 ("Riemenschneider"), mes. 1-4. Marche de type no 4 (a) et marche de type no 9 (b). 

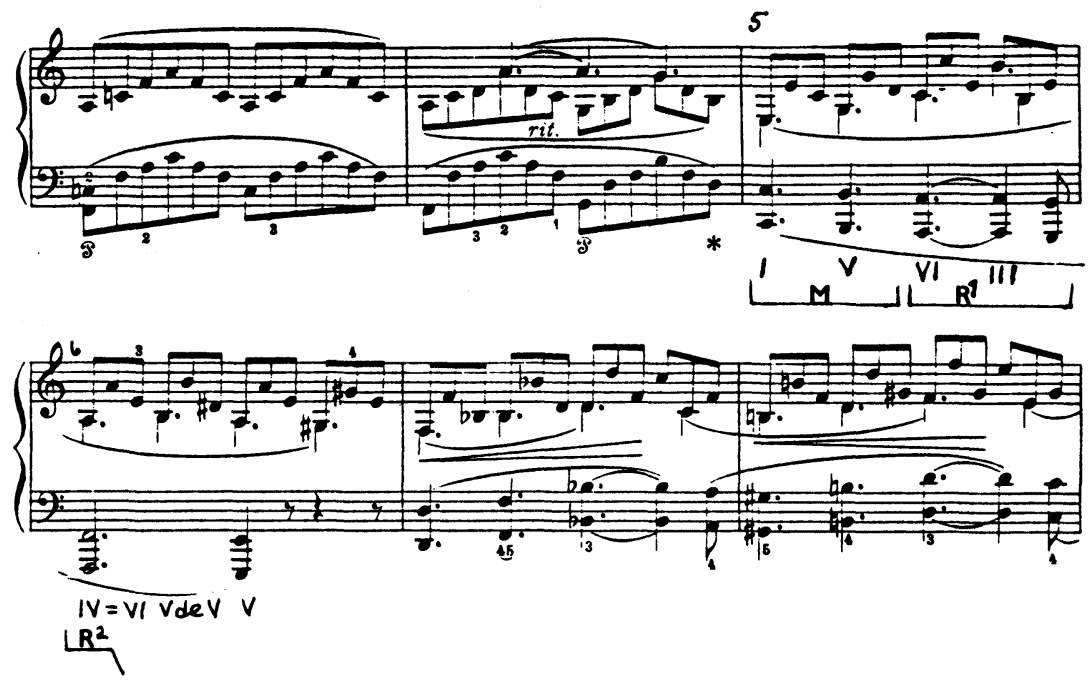

Figure 8. : Schumann : Fantaisie op. 17, III, mes. 5-6. Marche de type no 4.

Enfin, une seule reproduction du modèle suffit pour conclure à une marche (revoir l'exemple 2).

Une fois la marche définie, comment la classer? Le discours harmonique tonal relève, à toutes fins pratiques, d'un couple qui transcende tout le système et qui en est à la fois le moteur et la fin : le couple dominante-tonique (V-I). ${ }^{4}$ C'est essentiellement dans cette perspective que se situe notre entreprise de classification. Toute marche d'harmonie va donc être perçue comme le produit d'une imitation du couple V-I pris comme objet de référence mais n'apparaissant pas nécessairement à l'intérieur de la marche.

4 C'est la démonstration à laquelle se livre R. F. Goldman dans son remarquable Harmony in Western Music (1965). 
L'imitation renvoie à ce couple,

- soit en sens droit (V-I) :

Figure 9.

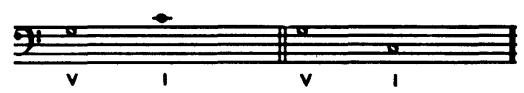

quel que soit par ailleurs le mouvement mélodique (geste de quarte ascendante ou de quinte descendante);

- soit en sens rétrograde (I-V) :

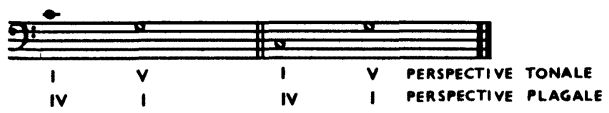

Figure 10.

Notons ici qu'une perspective plagale peut alors se substituer à la perspective tonale 5 (voir exemple 72 );

- soit en combinaison de ces deux sens (I-V-I) :

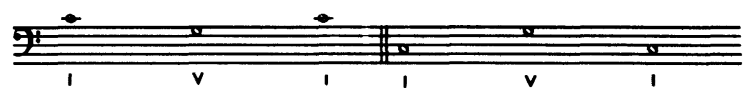

Figure 11.

5 Le discours harmonique tonal intègre plusieurs gestes plagaux, rappelons-le. 
Cette contrainte écarte de la classification non seulement les marches présentant plus de 3 accords dans le modèle mais également celles dont le modèle (à 2 ou à 3 accords) ne correspond pas au couple de référence. Un autre clivage apparaît ainsi. En effet, ces marches, de loin les moins fréquentes, s'alimentent à même des fragments de discours stéréotypé (matériel harmonique préfabriqué) que nous avons désignés plus haut comme étant des formules. ${ }^{6}$ Le modèle de la marche correspond alors à l'une ou l'autre de ces formules, par exemple :

III-II 6 -V-I = III...
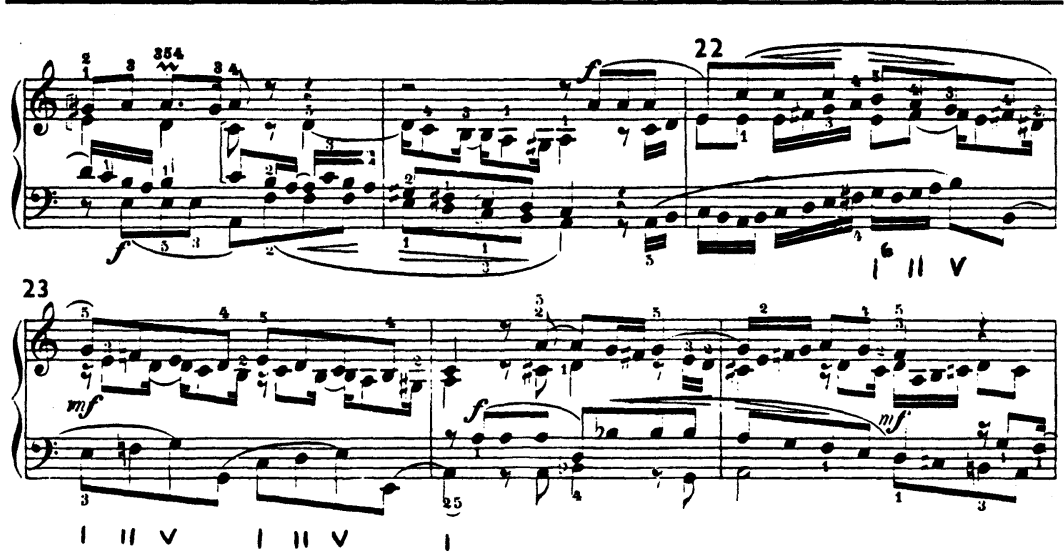

Figure 12. : J. S. Bach : Petits préludes et fugues, "Fugue no 8", mes. 22-24.

6 Une recension de ces formules fait partie intégrante du cours d'analyse donné par l'auteure sur le discours harmonique tonal. 
III-IV-V-I = III . .
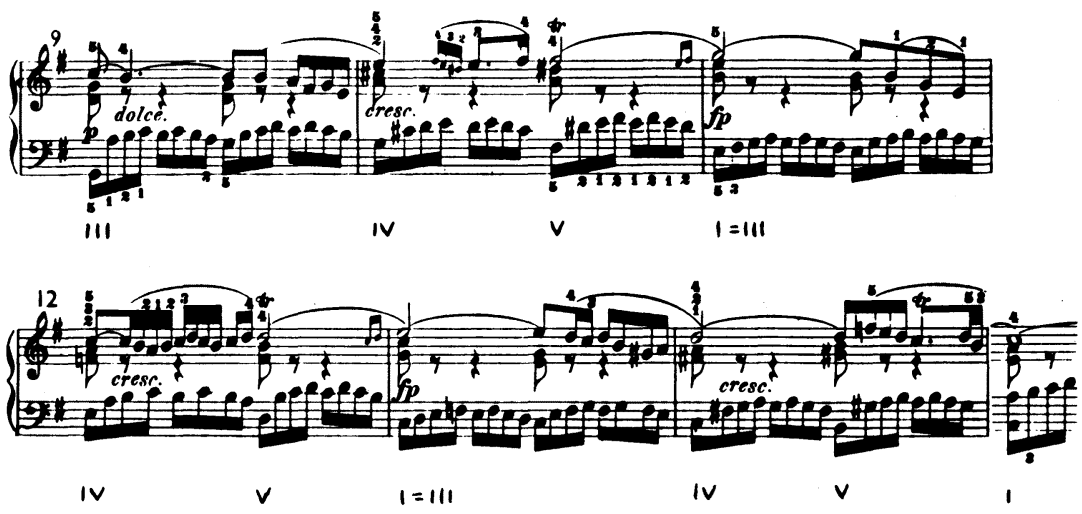

Figure 13. : J. S. Bach : Clavier bien tempéré, vol. 1, "Prélude no 10", mes. 9-15. 
$\mathrm{IV}-\mathrm{V}-\mathrm{I}=\mathrm{IV} \ldots$
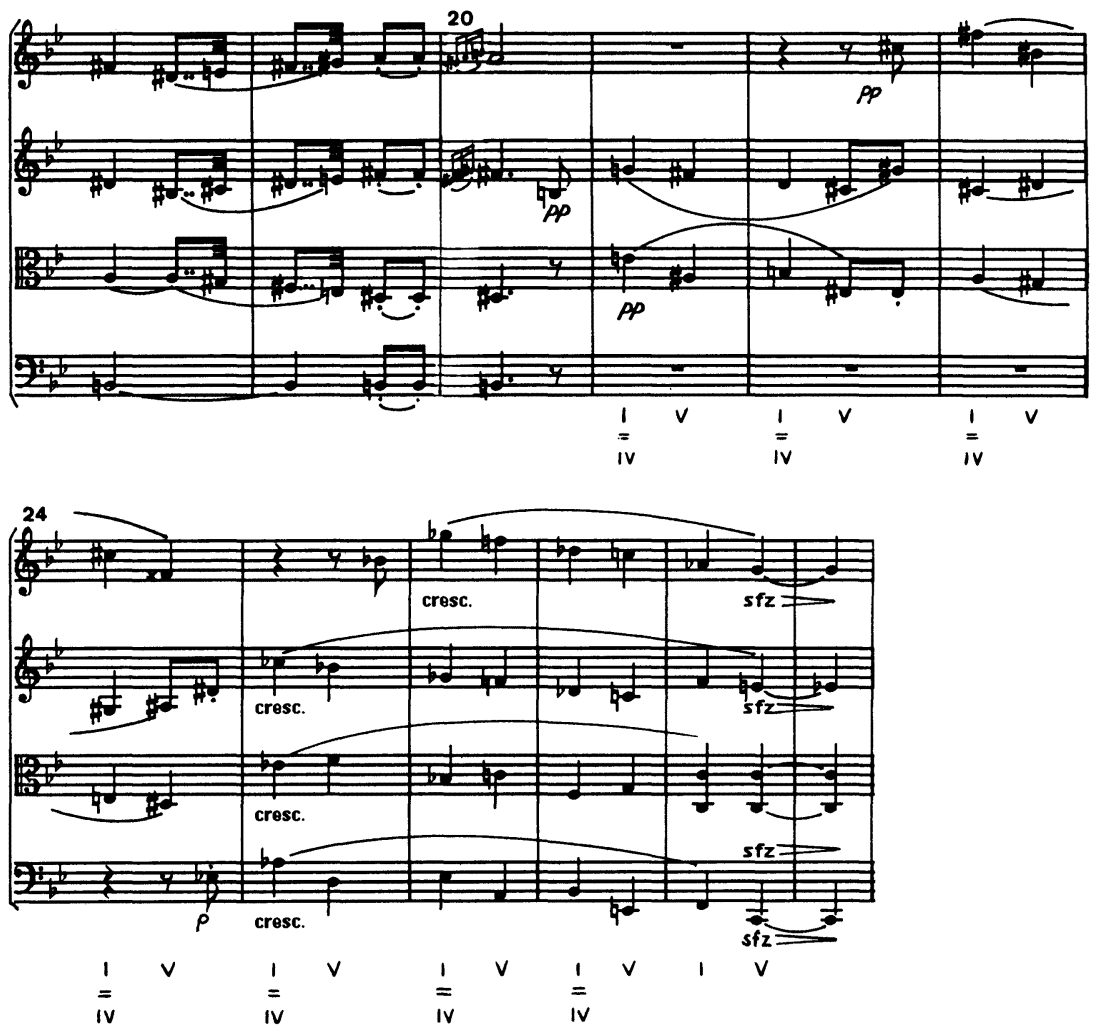

Figure 14. : Beethoven : Quatuor op. 18, no 6, IV, mes. 21-28. 
Le modèle peut même être constitué de plusieurs formules :
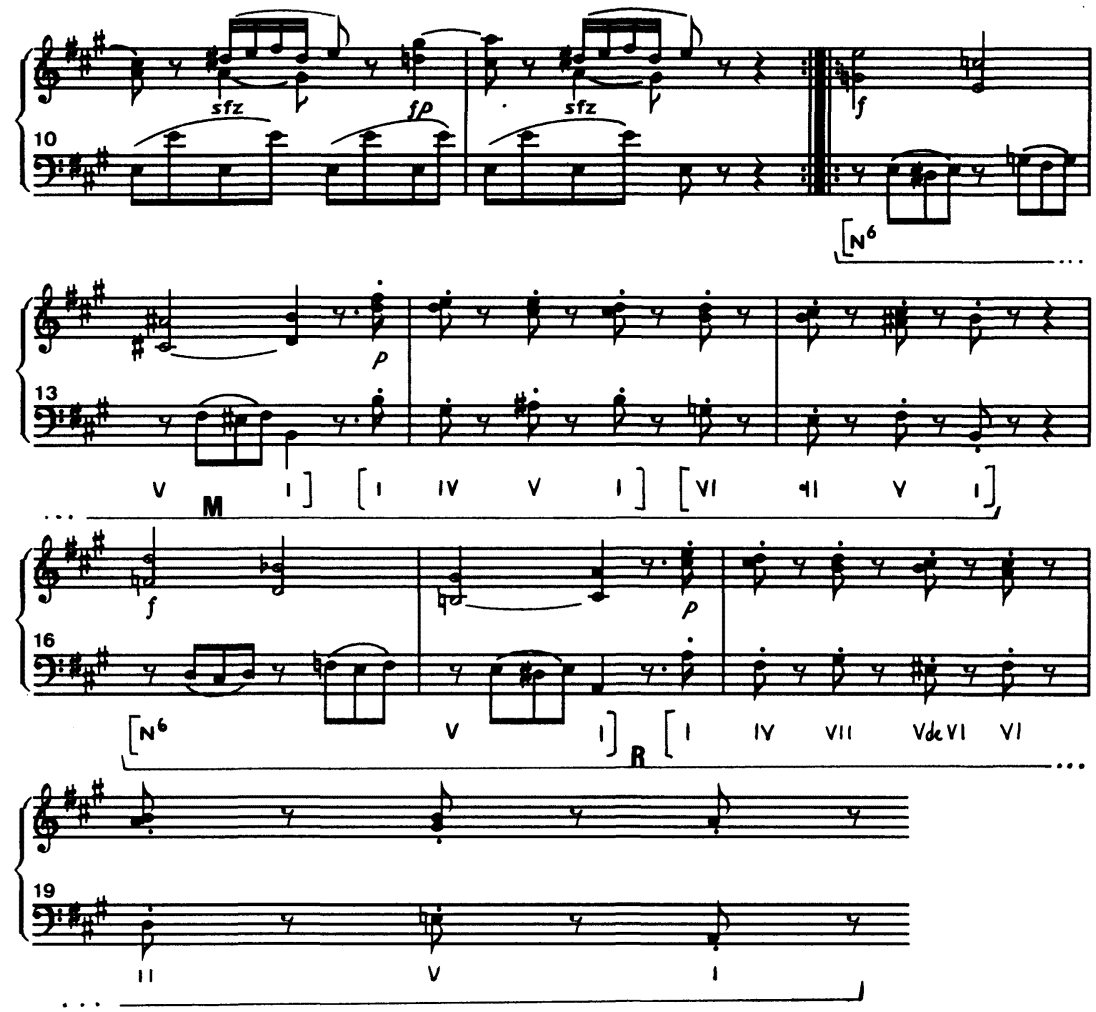

Figure 15. : Mozart : Sonatine viennoise no 2 (K. 439), I, mes. 12-19.

Noter ici que la reproduction, au lieu de répéter les 3 formules du modèle, réunit les 2 dernières en un seul ensemble (qui correspond à une marche de type no 1 , comme on le verra plus loin). 
À l'exclusion de ces cas, la classification proposée regroupe, sinon toutes, du moins la plupart des autres marches qui apparaissent dans le discours de Bach à Wagner. Trois critères de classification permettent d'en dégager 12 différents types :

1. Le jeu des fondamentales (réelles) dans le modèle.

Il faut préciser ici que l'on admet, dans le rôle de dominante à l'intérieur du couple V-I (principal ou secondaire), au moins 7 structures d'accords que voici :

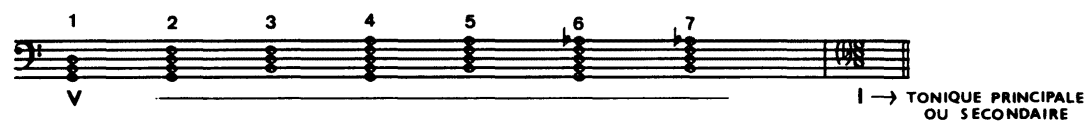

Figure 16.

C'est dire que les structures 3,5 et 7 ont comme fondamentale réelle une note non exprimée; il faut ajouter aussi que la quinte (à partir de la fondamentale réelle) de chacune de ces structures peut être altérée, soit haussée, soit abaissée d'un demi-ton.

2. La nature de l'intervalle de progression du modèle harmonique (seconde ou tierce).

3. La direction de l'intervalle de progression du modèle harmonique (marche descendante ou ascendante). 
Les 12 types de marche se répartissent alors selon le tableau suivant :

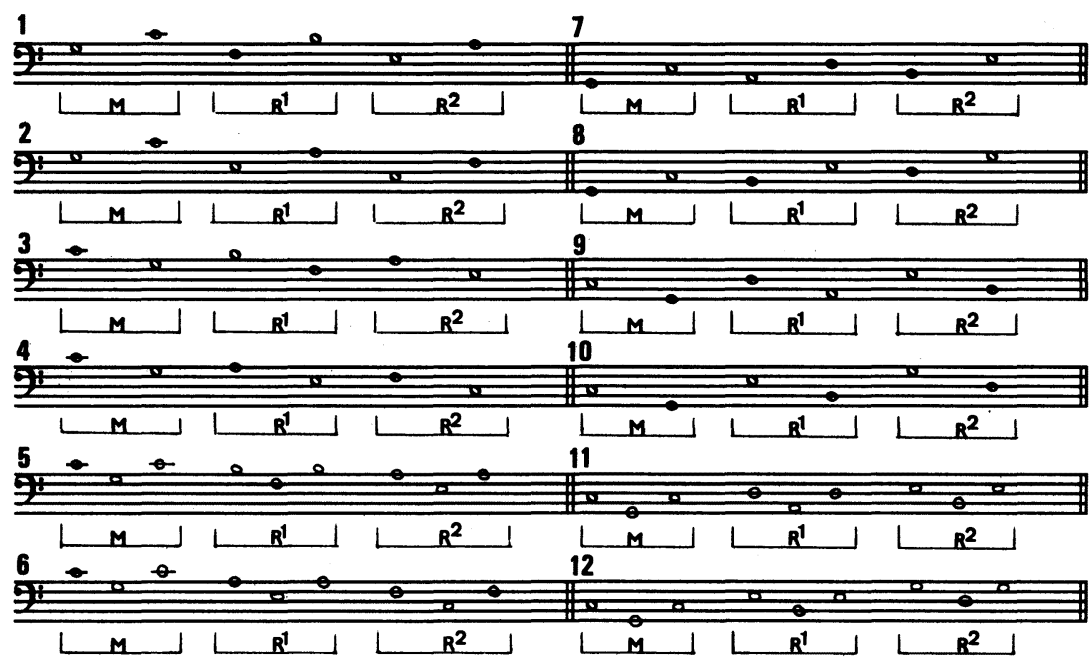

Figure 17. : Tableau de classification en 12 types des marches d'harmonie de Bach à Wagner

Il importe de signaler immédiatement que la classification d'une marche comme descendante (marches nos 1 à 6) ou comme ascendante (marches nos 7 à 12) relève d'un procédé d'abstraction qui lie le classement à la séquence des fondamentales (ainsi : do-fa-si- mi-la-ré-sol-do = marche descendante), de sorte que l'orientation mélodique descendante ou ascendante du discours lui-même, dans l'une de ses composantes ou dans toutes - à cause, par exemple, d'un geste d'octaviation -, peut sembler en contradiction avec le classement : 


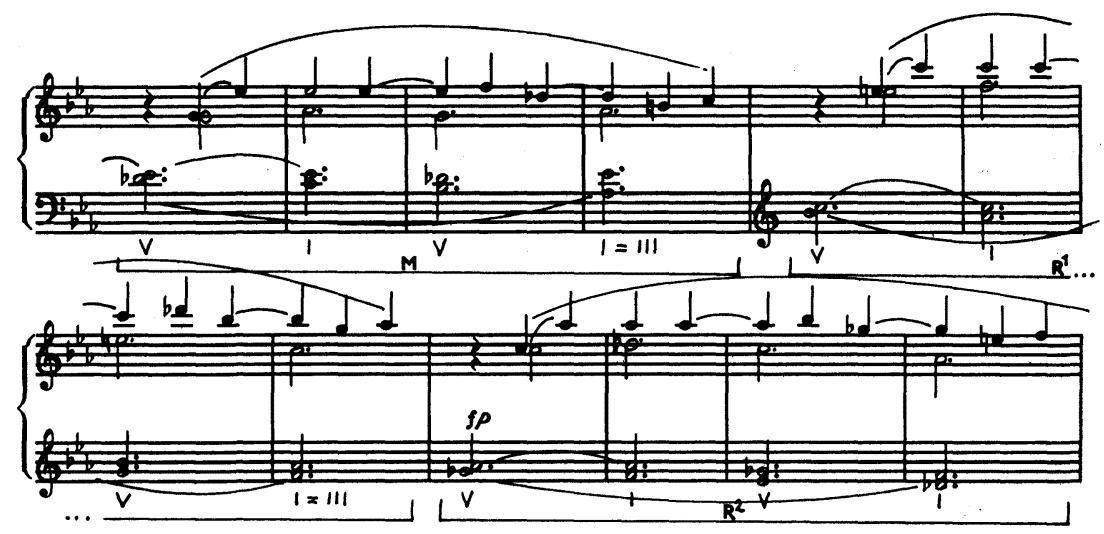

Figure 18. : Beethoven : Sonate op. 10, no 1, I, mes. 33-44. Marche classée descendante (de type no 2).

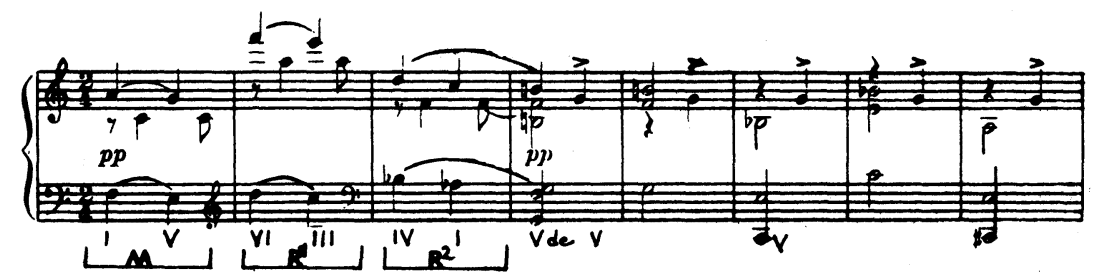

Figure 19. : Schubert : Symphonie no 7, II, mes. 1-4. Marche classée descendante (de type no 4). 


\section{FORMULATION MÉLODIQUE : LES MOTIFS}

Classer pour repérer, avons-nous dit. Or, ce qui accroche l'oreille ou retient l'oeil, c'est avant tout le jeu des lignes générées par les voix extrêmes de la marche harmonique. La formulation mélodique de ces 12 types de marche varie certes infiniment, en fonction, particulièrement, du contenu harmonique. Mais pas de n'importe quelle façon. Elle se réalise et se diversifie à partir de motifs caractéristiques qui, eux, ressortissent à des stéréotypes de conduite des voix lors du passage d'un accord à l'accord suivant. Ces motifs se prêtent à l'invention mélodique comme une sorte de squelette ou d'armature. Pour l'illustrer, on peut s'en tenir aux quatre types de marche présentant le plus fort taux de récurrence à travers le discours Bach-Wagner, soit les marches de type nos $1,4,7$ et 8 .

\section{a. Motifs caractéristiques de la marche de type no 1}

Voici la formulation la plus simple (exprimée à quatre voix) de cette marche, ${ }^{7}$ ainsi que le motif mélodique caractéristique qui, combiné à la voix de basse, servira à la repérer - ce motif étant énoncé le plus souvent à la voix supérieure mais pouvant apparaître aussi à d'autres voix :

7 Le lecteur désireux de se familiariser avec la marche de type no 1 pourra se reporter à la "Chaconne" de la Partita no 2 (ré mineur) pour violon seul de J. S. Bach, dont elle est la trame. 

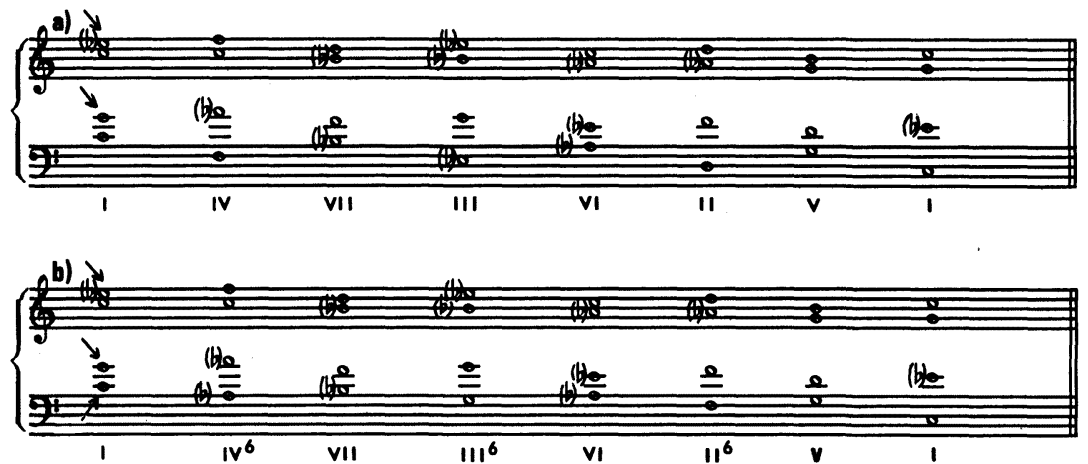

Figure 20.

- Ce premier motif mélodique se ramène ainsi à une ligne brisée, observable aux voix de soprano et de ténor dans l'exemple 20a), et en outre à la basse dans l'exemple 20b).

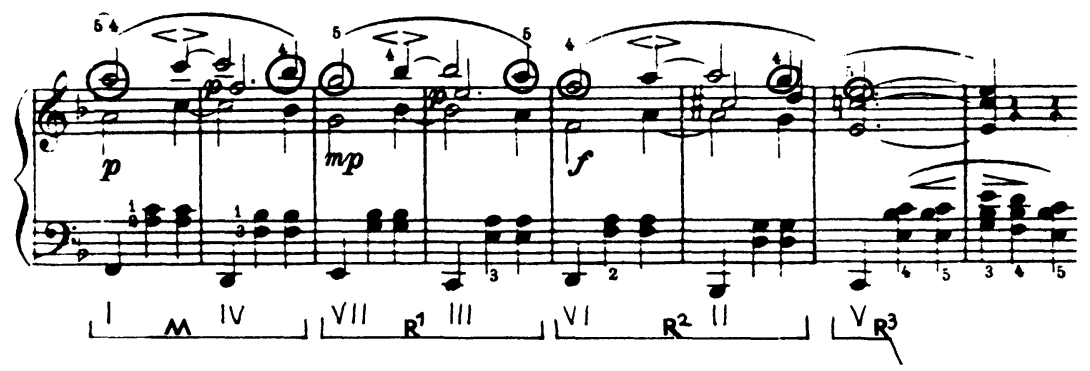

Figure 21. : Schumann, Op. 124, no 4, mes. 21-28. Marche de type no 1. 


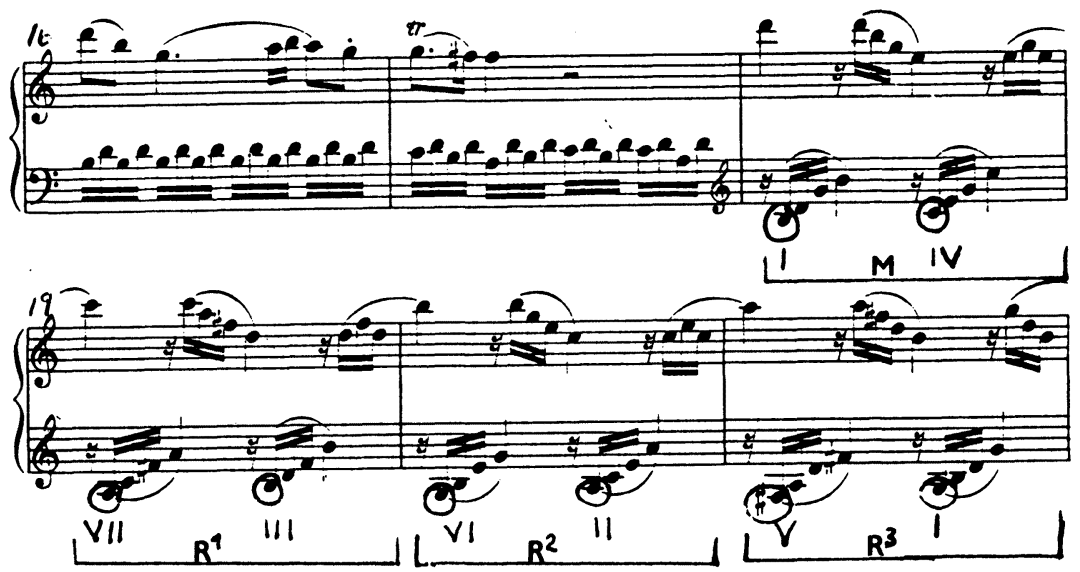

Figure 22. : Mozart : Sonate no 3 (K. 545), I, mes. 18-21. Marche de type no 1.

- L'intervention de dominantes secondaires fait apparaître un deuxième motif mélodique, soit une gamme chromatique descendante progressant à chaque accord :
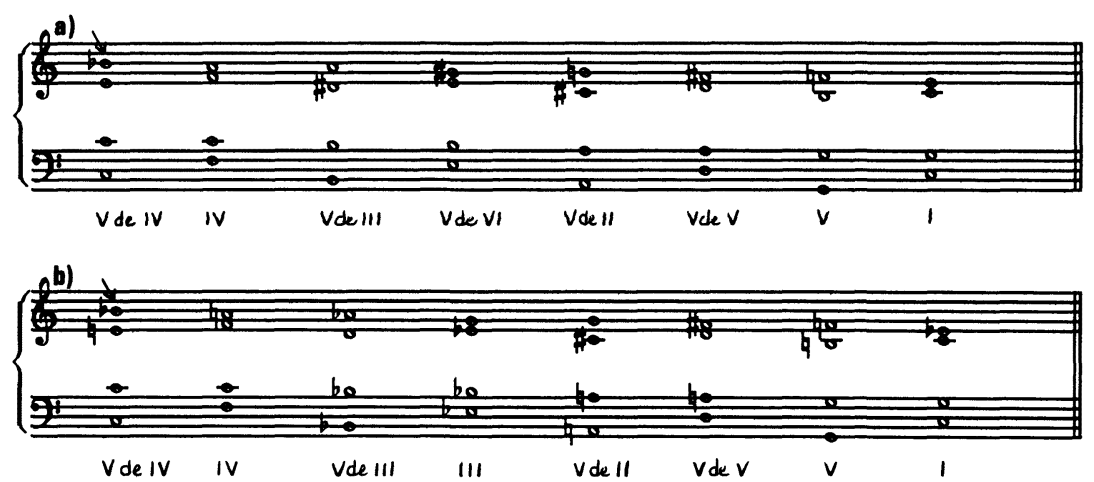

Figure 23. 


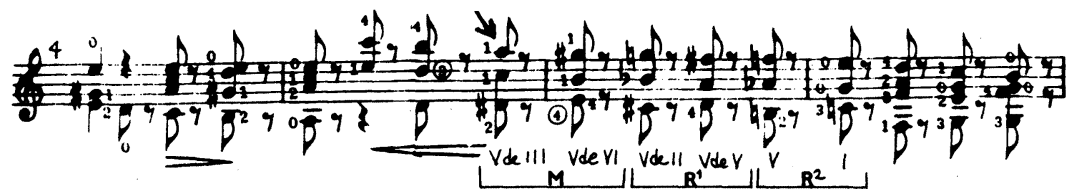

Figure 24. : Sor : Étude pour guitare op. 31, no 22, mes. 5-7. Marche de type no 1.

- Un troisième motif naît de l'intervention d'accords de septième, soit une gamme diatonique descendante progressant à tous les 2 accords :

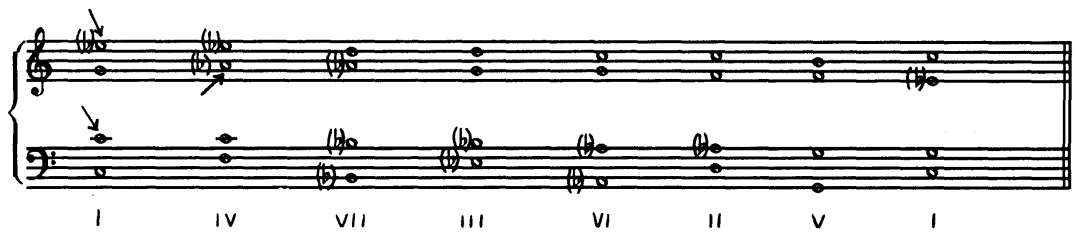

Figure 25. 

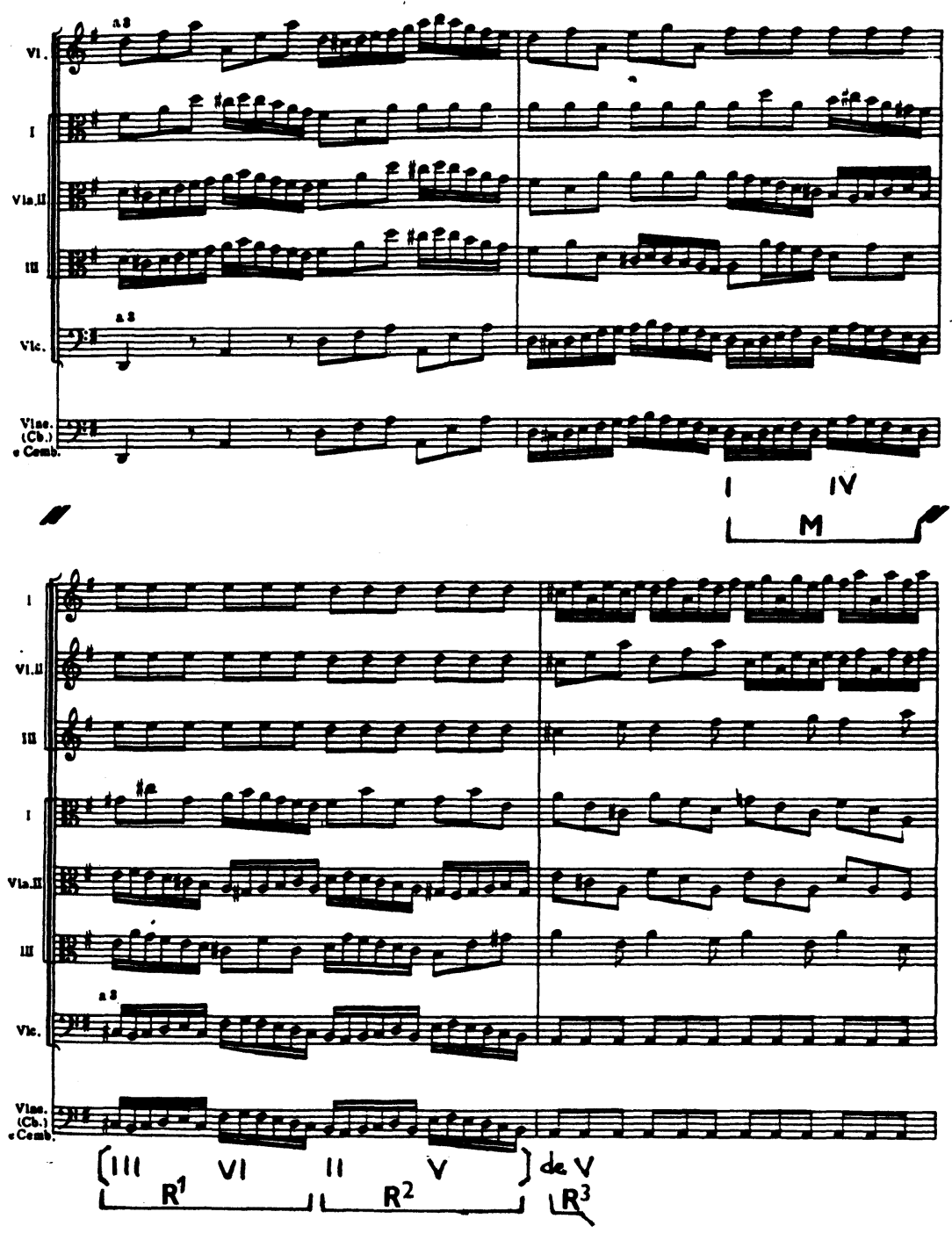

Figure 26. : J. S. Bach : Concerto brandebourgeois no 3, II, mes. 6-7. Marche de type no 1. 
Le texte suivant juxtapose à la voix supérieure les deuxième et troisième motifs :
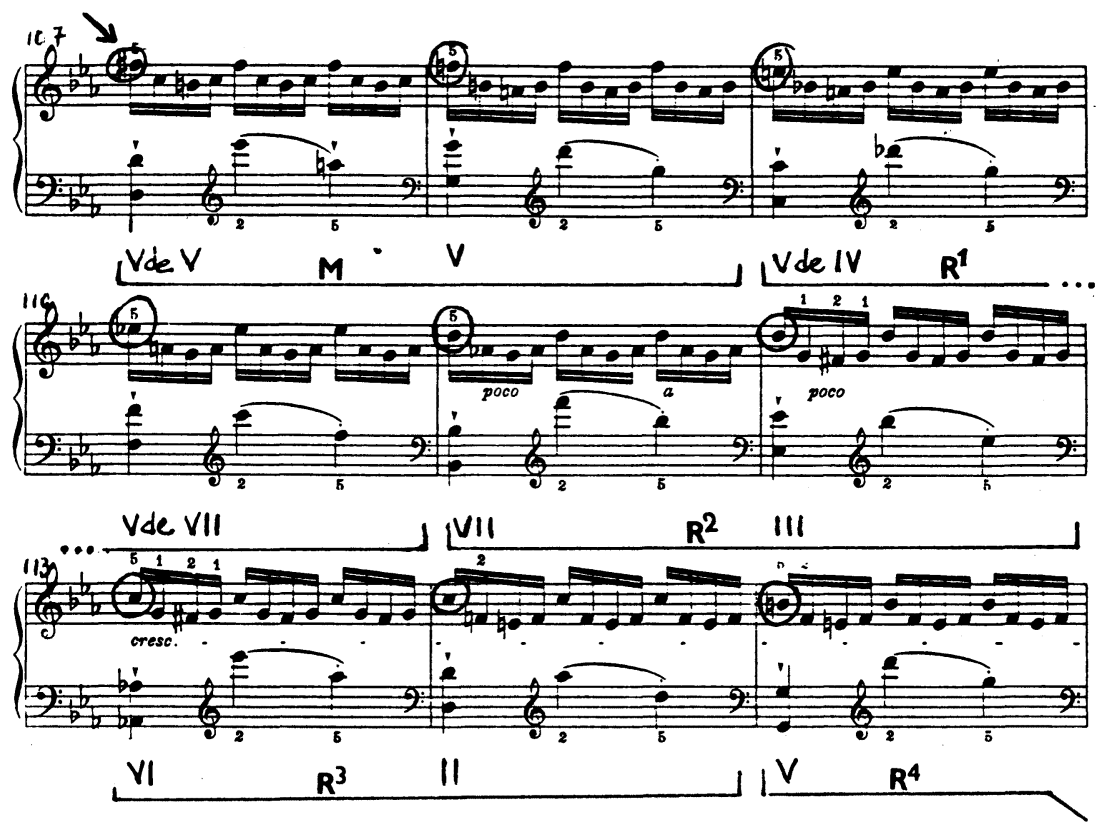

Figure 27. : Haydn : Sonate no 25, I, mes. 107-115. Marche de type no 1 .

\section{b. Motifs caractéristiques de la marche de type no 7}

La formulation de cette marche - sorte de pendant ascendant de la marche de type no 1 - se greffe à son tour sur 3 motifs principaux,

- soit une gamme diatonique ascendante progressant à tous les 2 accords : 


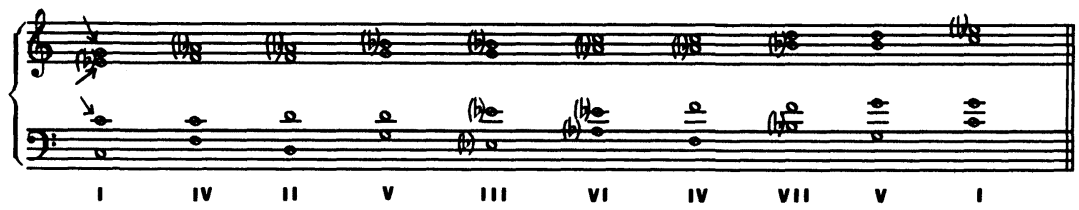

Figure 28.
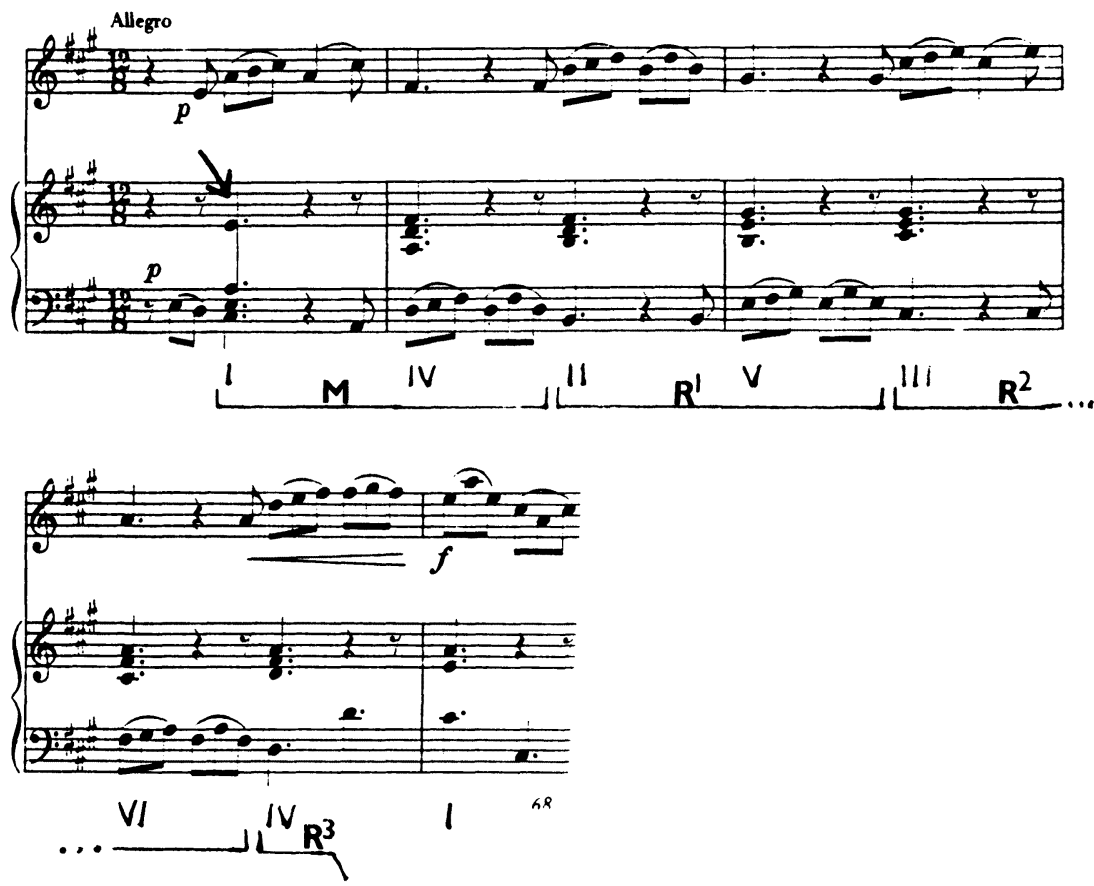

Figure 29. : Corelli : Sonate pour violon op. 5, no 9. Marche de type no 7 . 
- soit une gamme chromatique ascendante, due à l'emploi de dominantes secondaires :
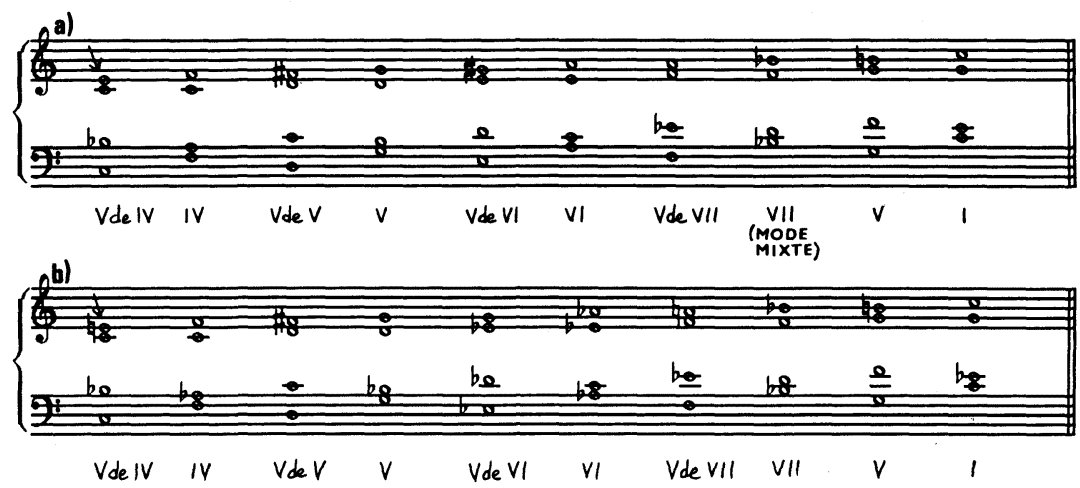

Figure 30. 
(Prelude.)
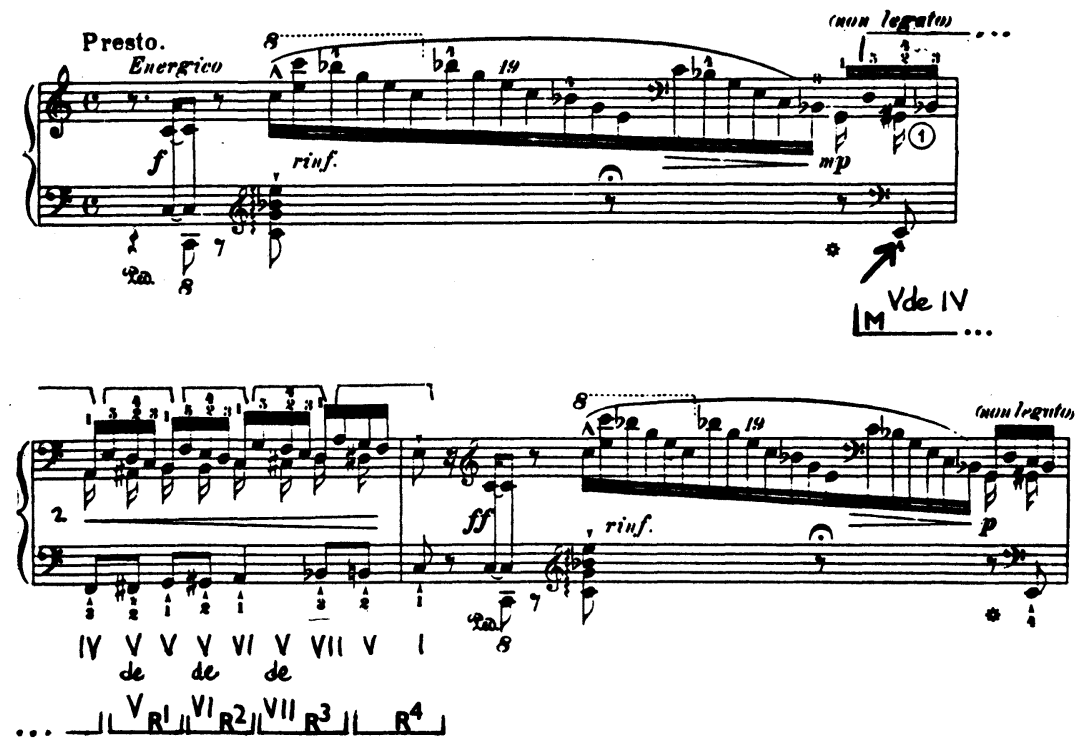

Figure 31. : Liszt : Étude d'exécution transcendante no 1, mes. 1-3. Marche de type no 1.

- soit un geste de tierce descendante, progressant à la quarte et provoqué par l'emploi systématique du mouvement contraire entre les voix supérieures et la voix de basse :

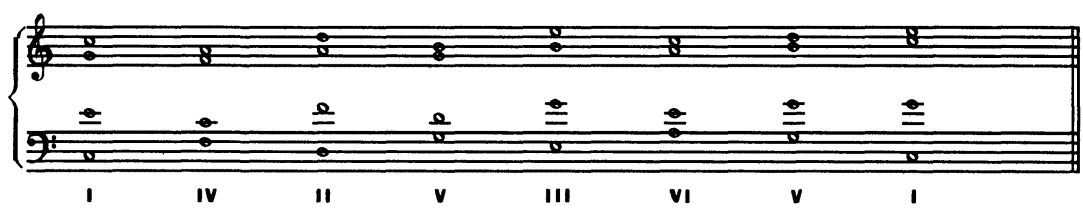

Figure 32. 

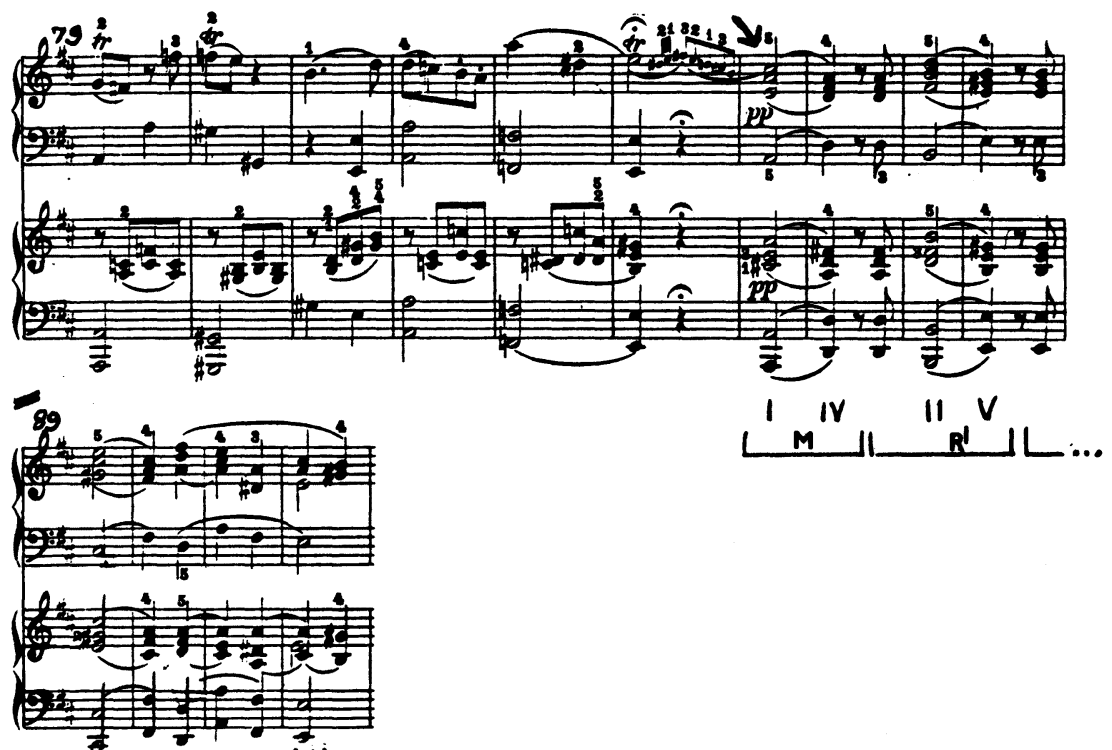

(14)

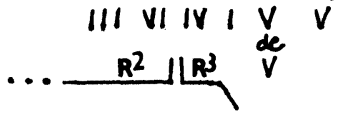

Figure 33. : Mozart : Sonate pour 2 pianos (K. 448), III, mes. 85-90. Marche de type no 7.

c. Motifs caractéristiques de la marche de type no 4

La réalisation de la marche de type no 4 présente, pour sa part, deux motifs contrastants. 
- Le premier motif renvoie à une gamme diatonique descendante progressant à chaque accord $:^{8}$

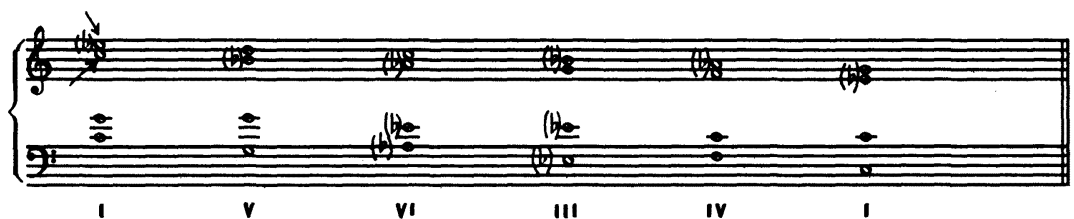

Figure 34.

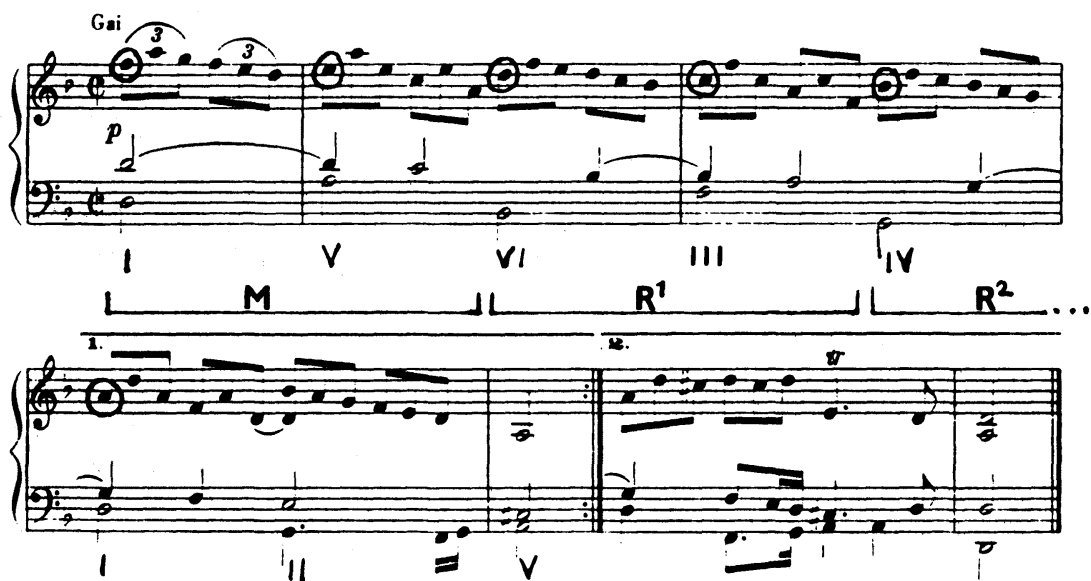

Figure 35. : Rameau : "Gavotte", Le temple de la gloire, Trio, mes. 1-4. Marche de type no 4.

8 Dans l'exemple 34, le lecteur reconnaîtra sans peine le canevas du trop célèbre Canon de Pachelbel. 
- Le second motif, en rapport avec l'emploi alterné d'accords à l'état fondamental et d'accords au premier renversement, aboutit à un geste de tierce, progressant à la tierce, charpente dont la facture particulièrement aérée se prête à une multitude de comblements :

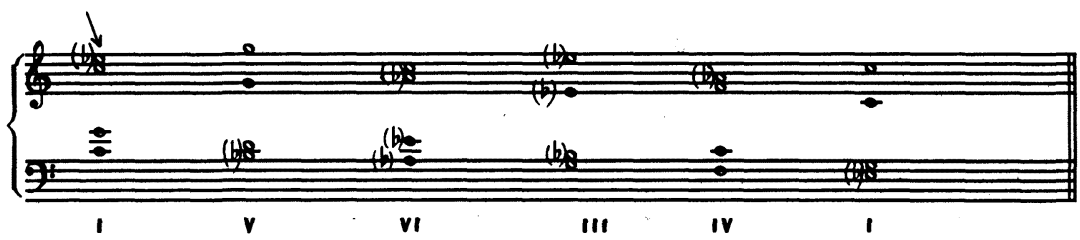

Figure 36.

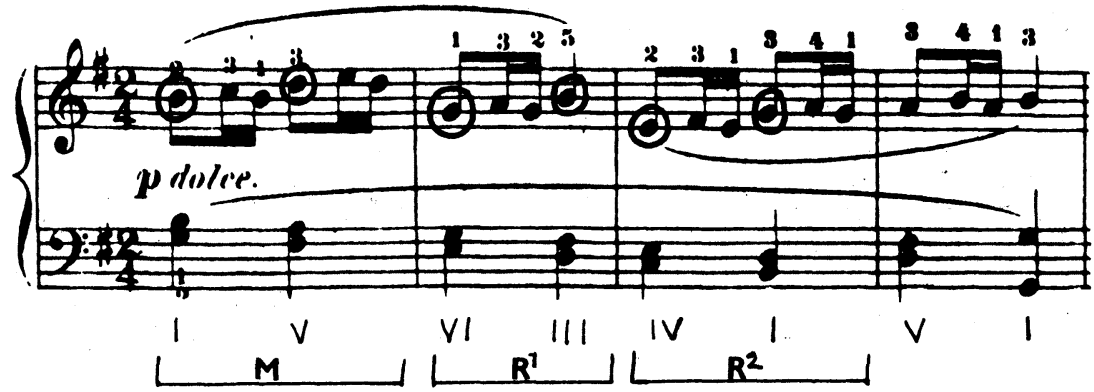

Figure 37. : Beethoven : Sonate op. 79, III, mes. 1-3. Marche de type no 4. 


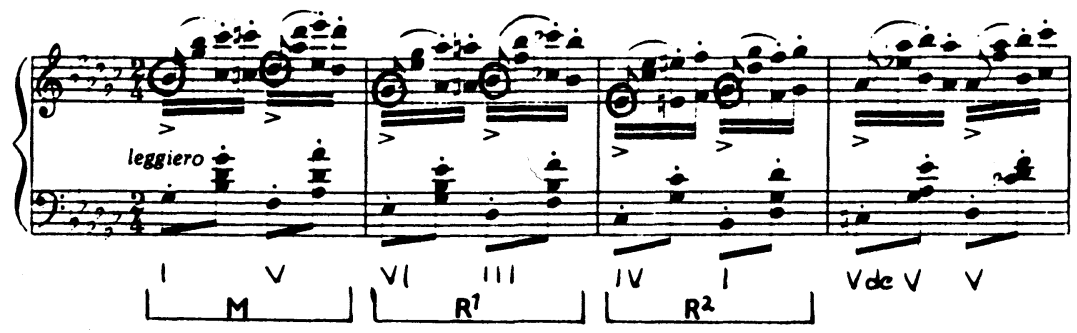

Figure 38. : Chopin : Étude op. 25, no 9, mes. 1-3. Marche de type no 4.

d. Motif caractéristique de la marche de type no 8

On ne retiendra, pour la marche de type no 8 , que le motif le plus courant, soit celui d'une gamme diatonique ascendante progressant à chaque accord :

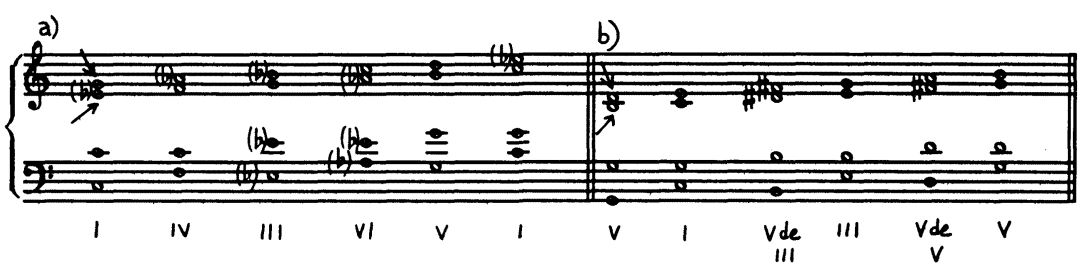

Figure 39. 

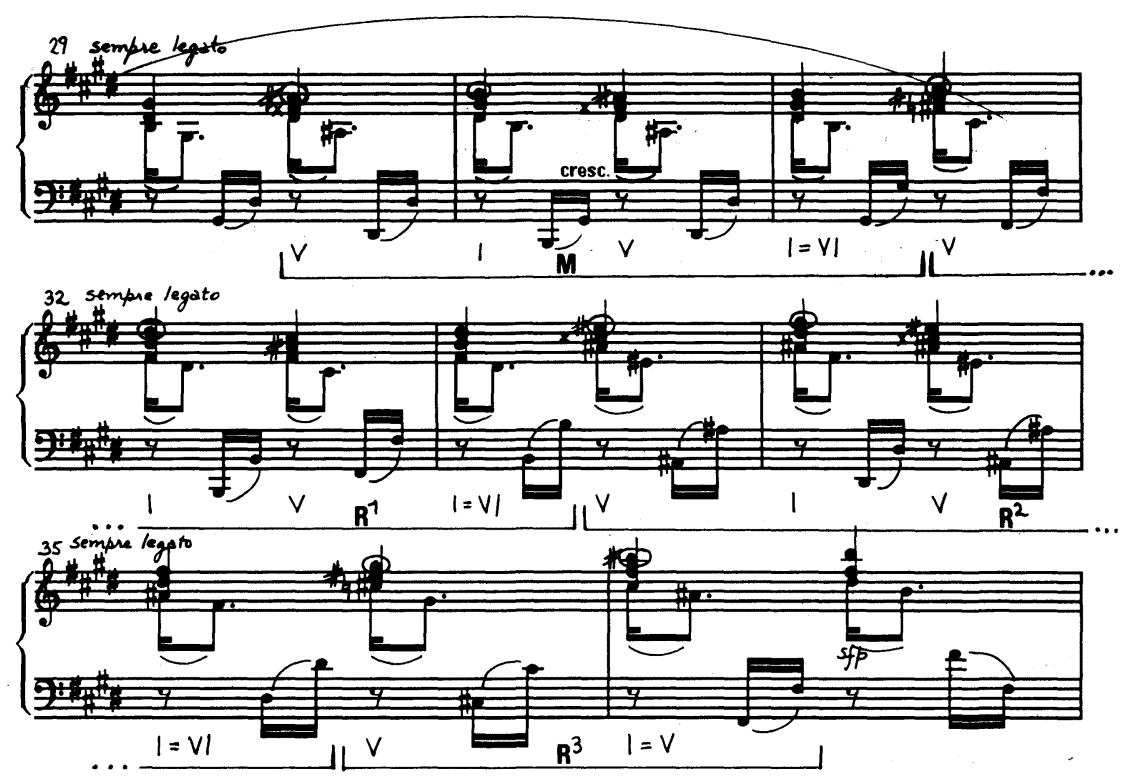

Figure 40. : Beethoven : Sonate op. 109, I, mes. 29-36. Marche de type no 8.

\section{L'ÊTRE TONAL DE LA MARCHE D'HARMONIE}

Le substrat mélodique des marches d'harmonie tonales, s'il s'avère fort utile pour le repérage, n'est cependant que partiellement indicatif de leur nature tonale lorsqu'on veut procéder à une analyse détaillée. "Être tonal" veut dire relever d'une hiérarchie, afficher une appartenance précise, se rattacher à un point d'ancrage dûment établi. Un même contenu de marche pourrait revendiquer plusieurs appartenances; ainsi : 


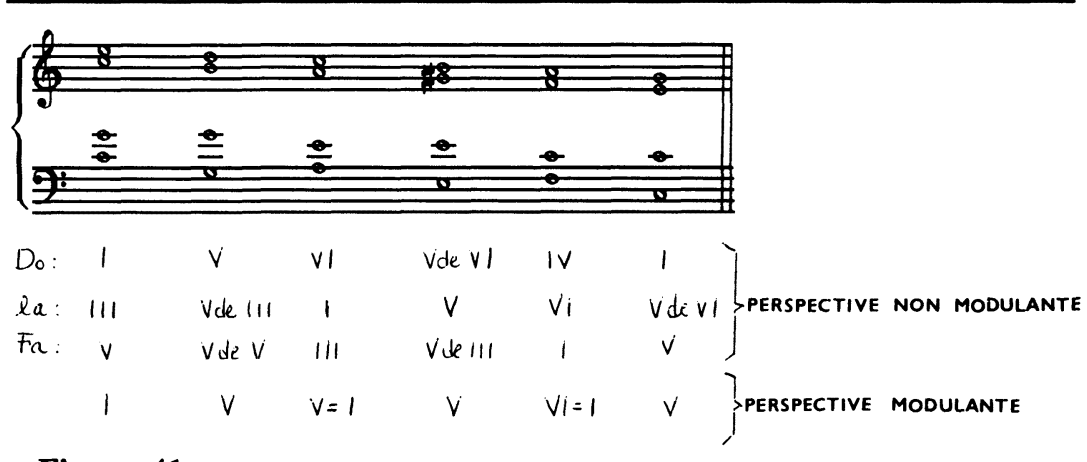

Figure 41.

C'est pourquoi la marche est habituellement close par une formule cadentielle qui en fixe la tonalité :

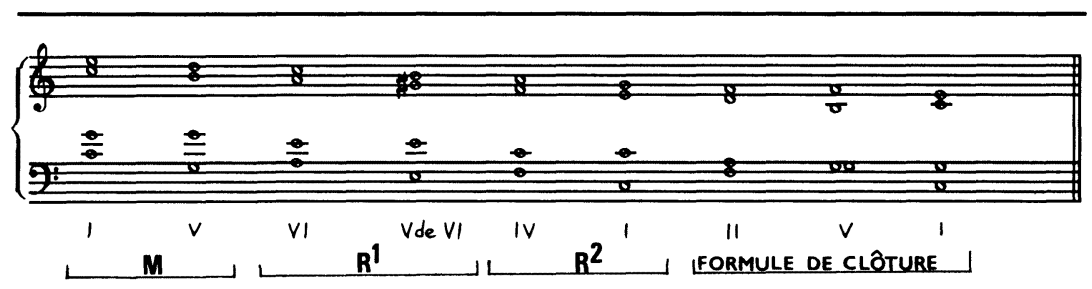

Figure 42.

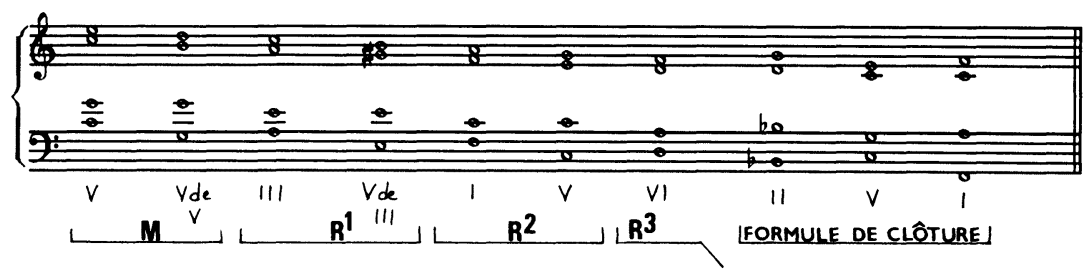

Figure 43. 


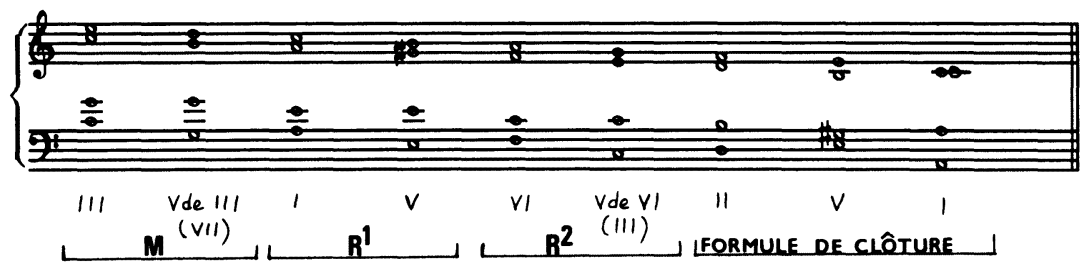

Figure 44.

À une exception près : la marche de type no 1 qui, se déroulant à même un cycle de quintes descendantes, se présente souvent sans formule de clôture et, en ce cas, voit automatiquement son point d'ancrage désigné dès l'arrêt du discours, qui survient au moment de la fonction I ou de la fonction $\mathrm{V}$ :

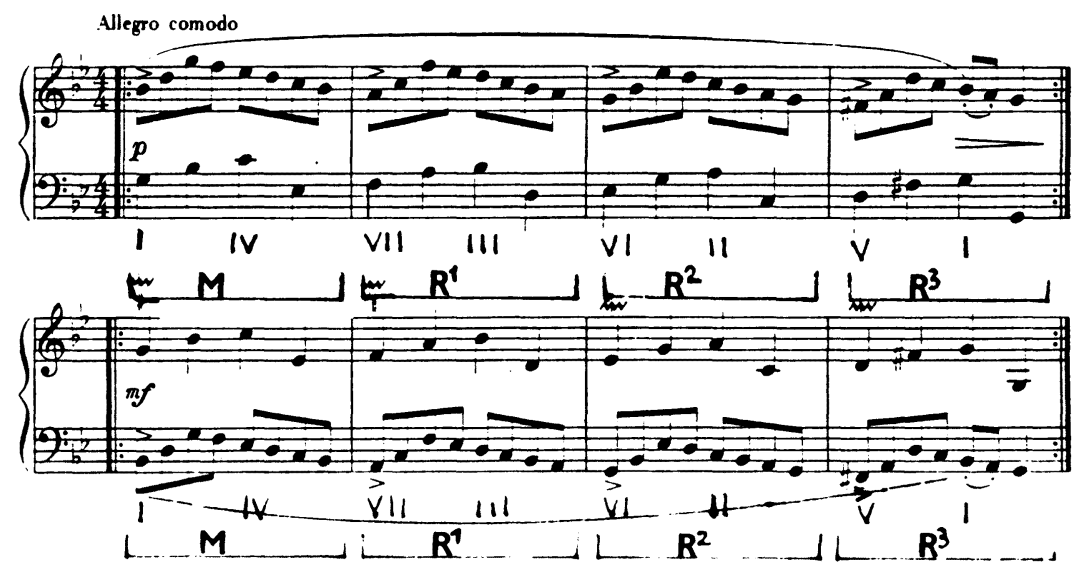

Figure 45. : Händel : Suite no 7, "Passacaille". Marche de type no 1 . 
On va donc, d'une manière générale, convenir au sujet de la marche d'harmonie d'une structure tripartite.

\section{STRUCTURE TRIPARTITE DE LA MARCHE D'HARMONIE}

1. Le modèle.

Il arrive que le modèle soit bissé (cf. exemples 17 et 39).

2. Une ou plusieurs reproductions de ce modèle.

Lorsqu'il y a plusieurs reproductions du modèle, la dernière d'entre elles peut n'être qu'amorcée, comme on a déjà pu l'observer.

3. La formule de clôture.

Ce rôle de désignation d'une tonalité est parfois tenu par l'avènement d'une autre marche qui partage ou non le même modèle. Dans l'affirmative, on observe en quelque sorte une structure de marche mixte imbriquant 2 types différents. Soulignons aussi que la formule de clôture, à moins de correspondre à une autre marche, se trouve en dehors du processus d'imitation. 


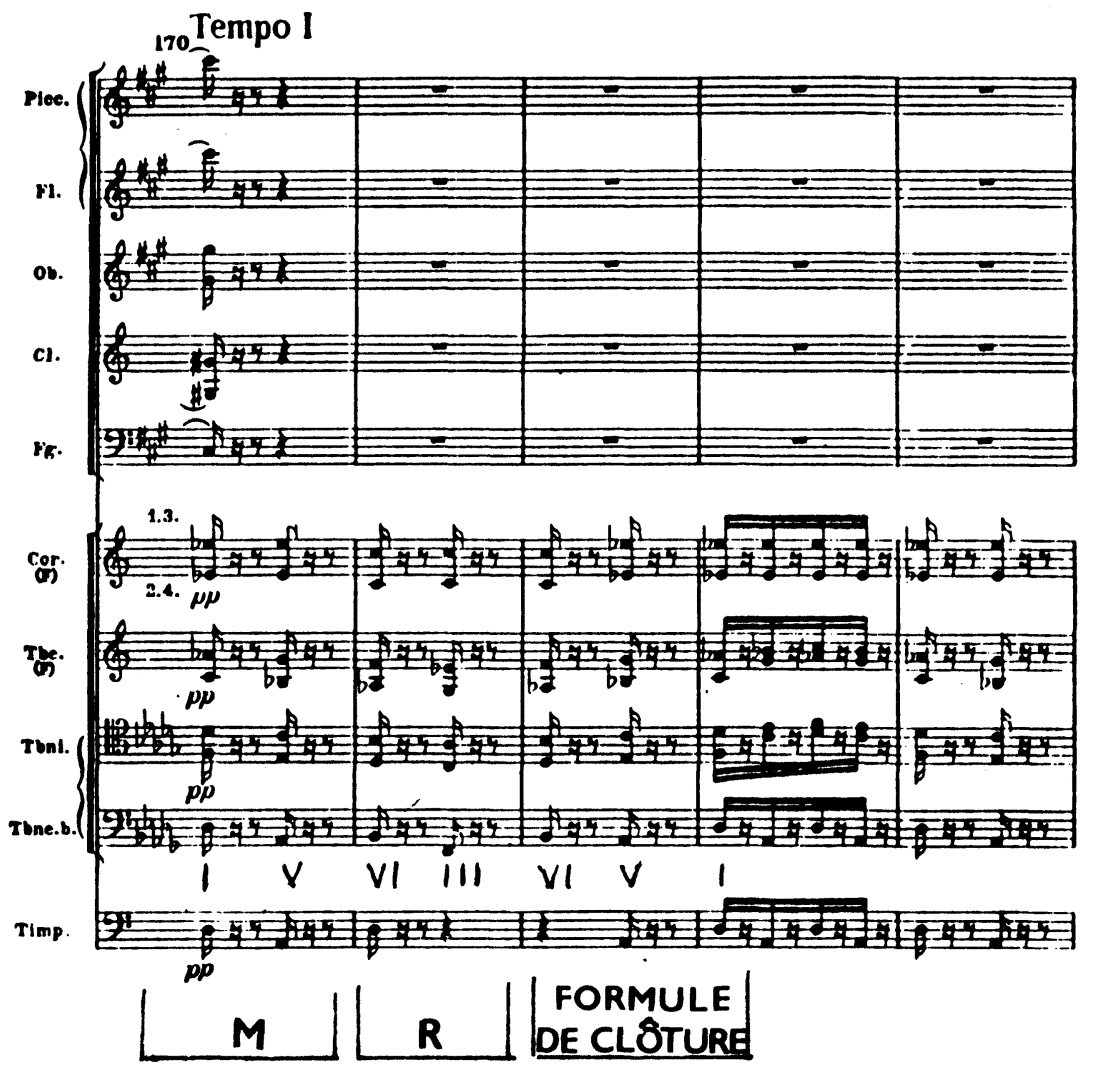

Figure 46. : Tchaikovsky : Symphonie no 4, op. 36, III, Trio, mes. 170-173. Marche de type no 4. 

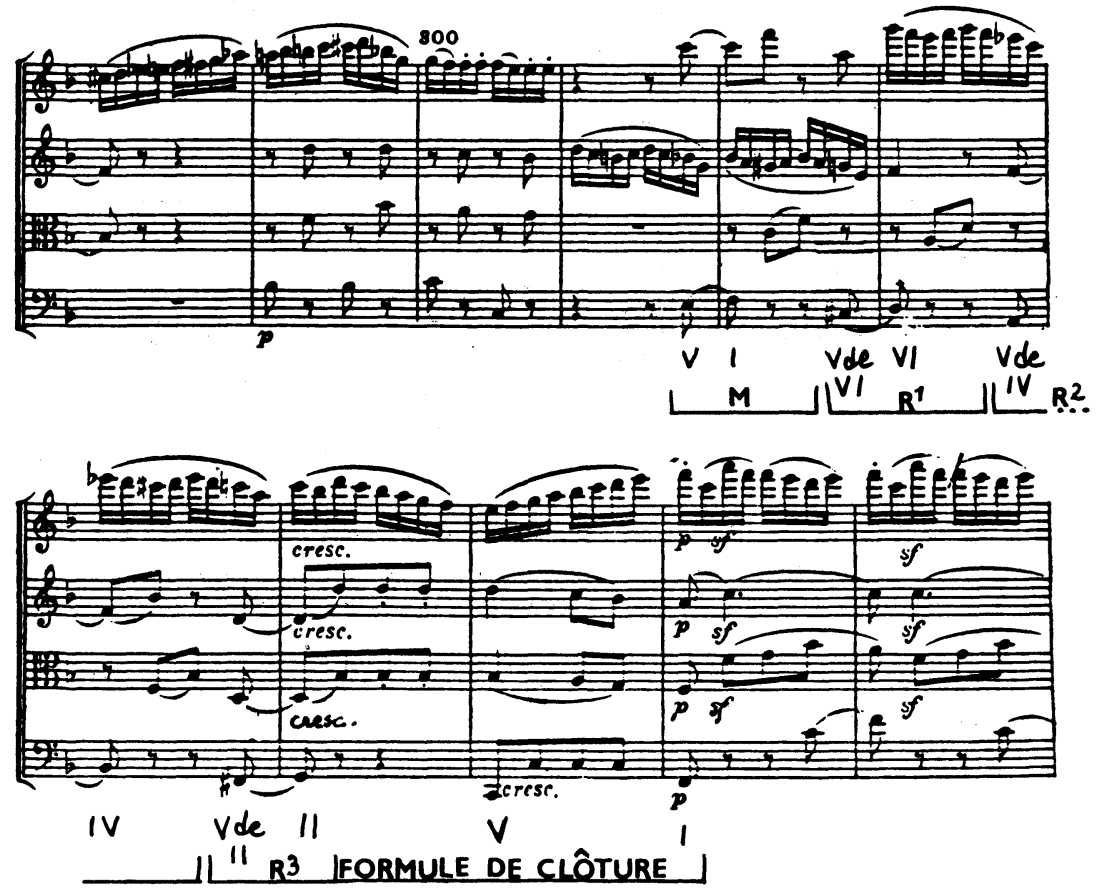

Figure 47. : Beethoven : Quatuor op. 18, no 1, IV, mes. 301-307. Marche de type no 2. 


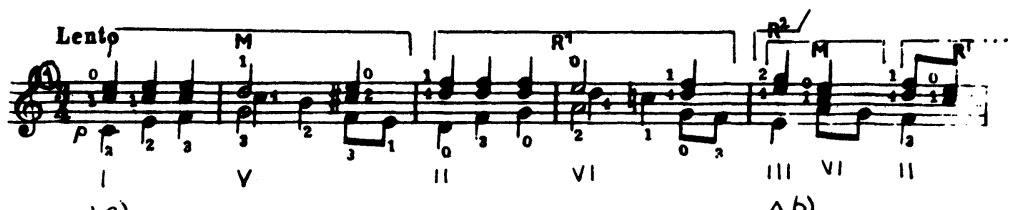

a)

(b)

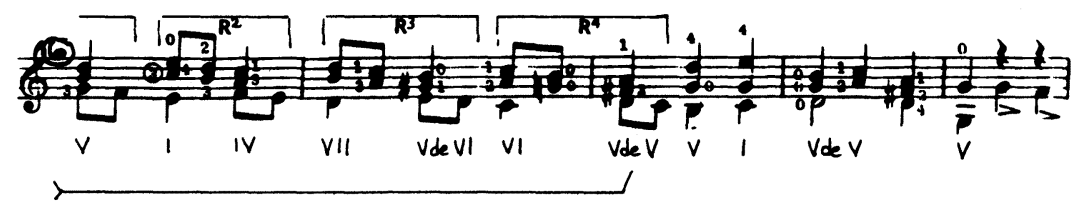

Figure 48. : Sor : Étude pour guitare op. 6, no 4, mes. 1-10. Marche de type no 9 (a) et marche de type no 1 (b).

D'autre part, une marche privée de sa formule de clôture pourra voir sa tonalité déterminée par le contexte : 

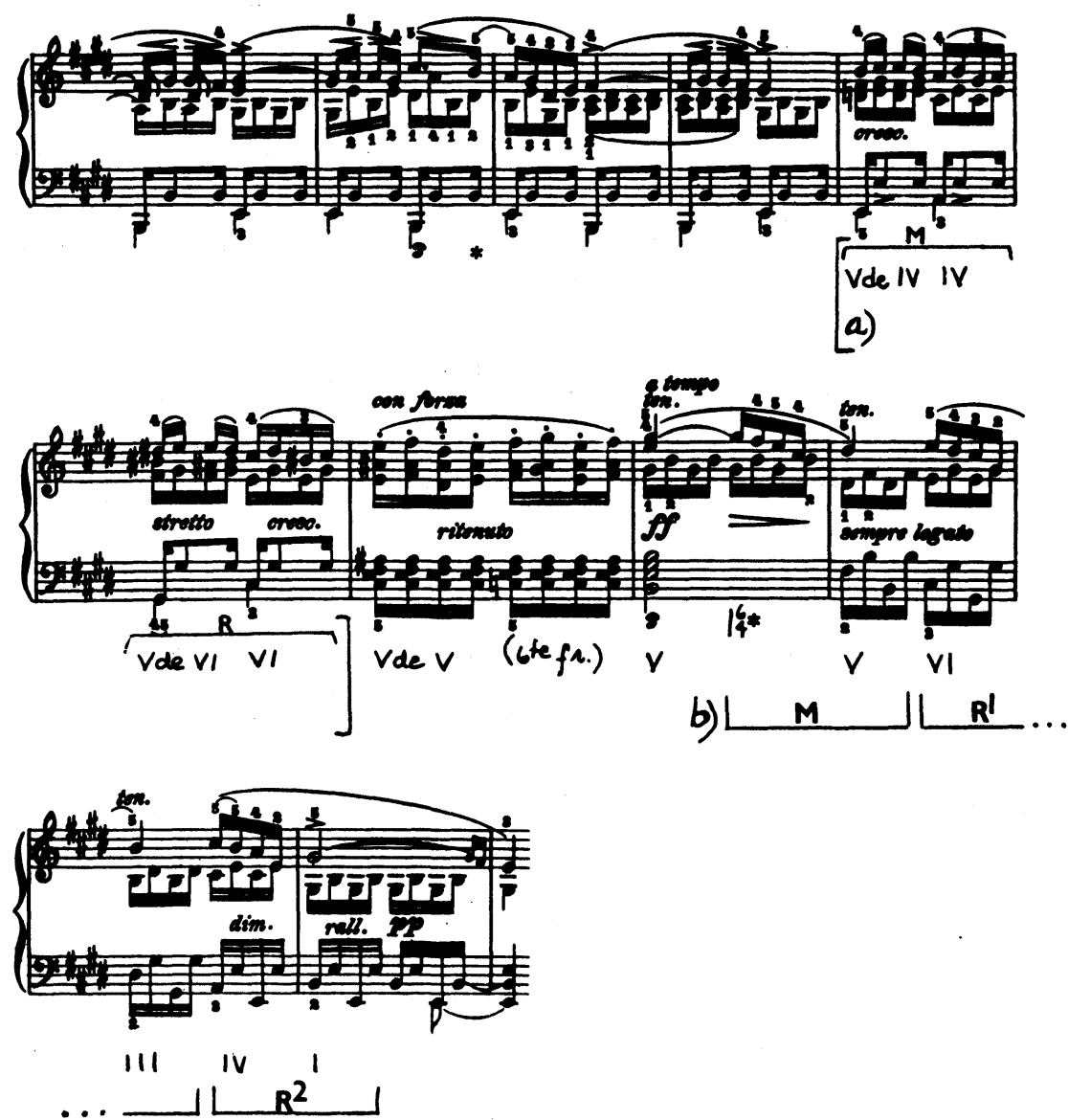

Figure 49. : Chopin : Étude op. 10, no 3, mes. 17-21. Marche de type no 4.

À partir du dernier exemple, il y a lieu de faire remarquer le pouvoir d'installation que détient la marche no 4 ainsi énoncée, 
privée de sa formule cadentielle. ${ }^{9}$ D'ailleurs, cette séquence, même isolée de tout contexte, s'entend spontanément comme I-V-VI-III-IV-I, rejoignant là une longue tradition d'emploi qui nous la montre déjà bien personnalisée dès le XVIe siècle.

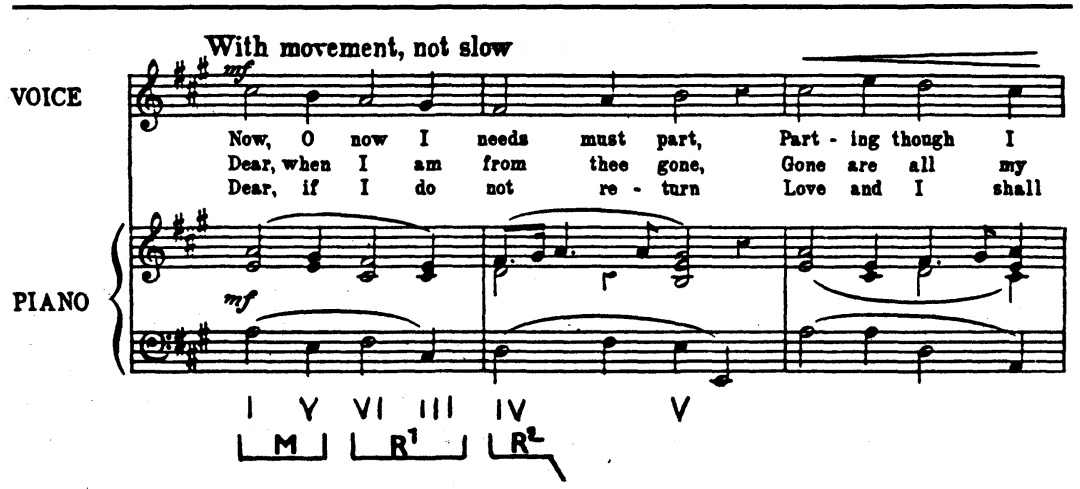

Figure 50. : J. Dowland : Now, O now I needs must part, mes. 1-2. Marche de type no 4.

\section{MARCHE MODULANTE OU NON?}

La formule de clôture ne saurait toutefois régler toute la question de l'appartenance tonale. Toute marche, de quelque type que ce soit, peut encore relever soit d'un traitement non modulant, soit d'un traitement modulant. Dès lors, le problème reste entier de la perspective à choisir dans le cas d'une marche pouvant s'inscrire aussi bien dans un discours non modulant que dans un discours modulant (revoir exemple 40). Pour y répondre de manière pertinente, plusieurs éléments doivent être pris en compte dont le premier seulement est intrinsèque à la marche alors que les autres en sont extérieurs :

9 Il conviendrait ici de considérer également la structure du conditionnement perceptuel tonal qui incite l'auditeur à faire coïncider la fin d'un événement harmonique avec une fonction de tonique si possible, dans l'expectative d'un événement achevé, accompli. 
1. Le comportement d'une marche modulante, qui a tendance à réaliser une parfaite symétrie dans la reproduction, tant au niveau des structures d'accord utilisées dans le modele qu'au niveau de l'intervalle de progression, par opposition à celui d'une marche non modulante qui, contrainte d'utiliser les accords constitutifs d'une tonalité et de progresser sur ses degrés, aboutit le plus souvent à une symétrie irrégulière (surtout lorsqu'elle comporte plus d'une reproduction du modele).

2. La mise en parallèle de la tonalité de l'événement précédant la marche avec la tonalité désignée par la formule de clôture à l'issue de la marche; la coïncidence des deux tonalités suggère une perspective de continuité.

3. Le contexte formel dans lequel s'insère la marche. Par exemple, une section de transition, une section de divertissement, ou une section de développement à l'intérieur de la forme sonate amènent à privilégier une perspective de modulation; tandis qu'une section d'exposition du matériau thématique appelle plutôt un régime de stabilité.

4. L'objectif d'uniformisation de la perspective d'analyse pour l'ensemble de l'oeuvre.

\section{CONTENUS HARMONIQUES DIVERSIFIÉS}

\section{a. Variantes de surface}

La pondération de ces quatre critères va s'avérer fort précieuse par rapport à la très grande diversification de contenu harmonique qui peut toucher chacun des 12 types de la classification.

La nature même de la marche de type no 1 qui, nous l'avons déjà souligné, circule à travers un cycle de quintes descendantes, structure elle-même considérée comme fondement du discours tonal, 10 en fait le

10 Encore là, nous renvoyons le lecteur à l'ouvrage de Goldman (1965) dont c'est la perspective centrale. 
champ d'activité, et donc d'observation, par excellence. Notre examen se limitera d'abord aux variantes de surface, soit de celles qui n'affectent pas le jeu des fondamentales. La marche de type no 1 sous l'angle modulant

Sous l'angle modulant, cette marche peut véhiculer plusieurs structures harmoniques différentes :

1. Un cycle de quintes (modulant) où l'on change de tonalité à chaque accord. L'événement se présente le plus souvent sous la forme d'une suite d'accords de $7 \mathrm{e}$ de dominante ou d'accords de $7 \mathrm{e}$ diminuée. En ce qui concerne le parallélisme d'accords de $7 e$ diminuée, il y aura toujours lieu d'envisager également, à son endroit, un statut ornemental excluant le mouvement harmonique; en cas d'alternative, on peut toujours recourir aux critères cités plus haut.

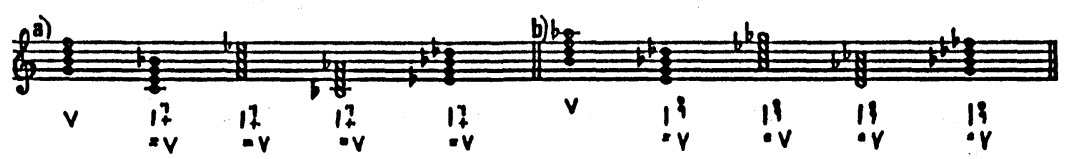

Figure 51. 

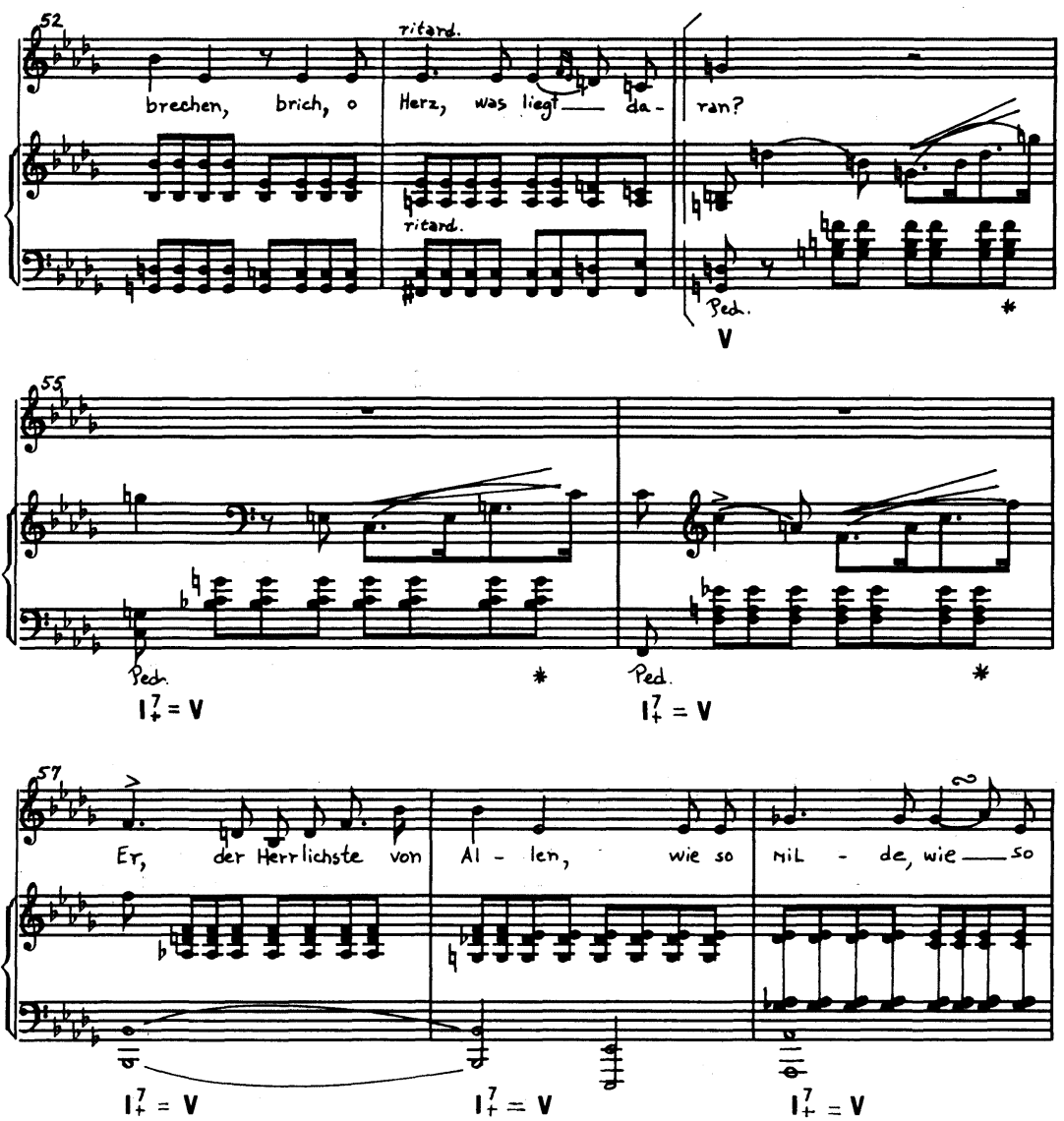

Figure 52. : Schumann : Op. 42, no 2, mes. 54-59. Marche de type no 1.

On verra très souvent apparaître cette structure (d'un cycle de quintes modulant), cependant amplifiée dans le temps et dans l'espace, à l'intérieur de la section de développement d'une forme sonate de facture classique simple. 
2. Des formules en marche progressant par tons ou demi-tons descendants. Par exemple, et parmi les plus courantes :

- $\quad$ II-V-I = II . . caractérisée par l'alternance d'un accord parfait mineur (seule structure habilitée à remplir à la fois une fonction de I et une fonction de II) et d'une structure pouvant jouer le rôle de $\mathrm{V}$ :

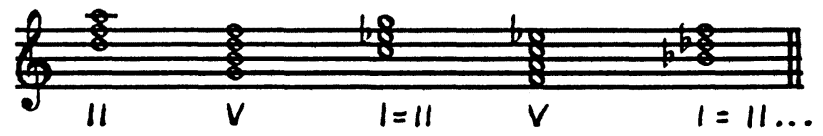

Figure 53.

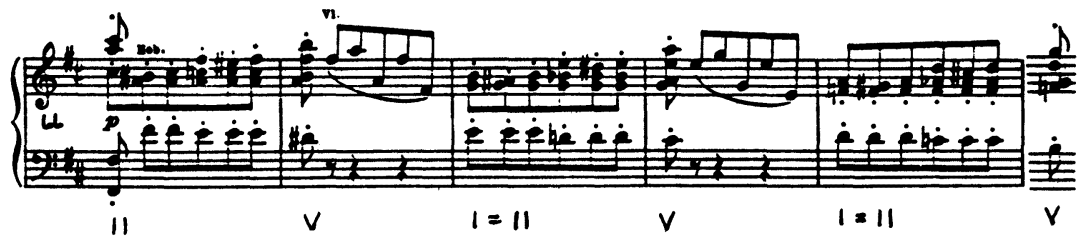

Figure 54. : Brahms : Symphonie no 2, I, mes. 66-71. Marche de type no 1 . 
- $\quad \mathrm{N}-\mathrm{V}-\mathrm{I}=\mathrm{N}$ (accord de sixte napolitaine)... caractérisée par l'alternance d'un accord parfait majeur et d'une structure pouvant jouer le rôle de $\mathrm{V}$ :

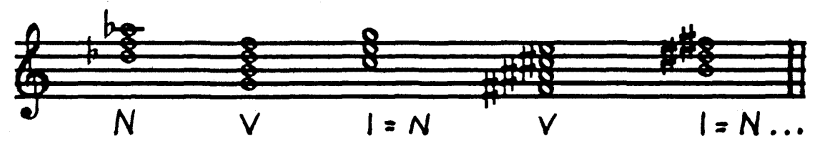

Figure 55.
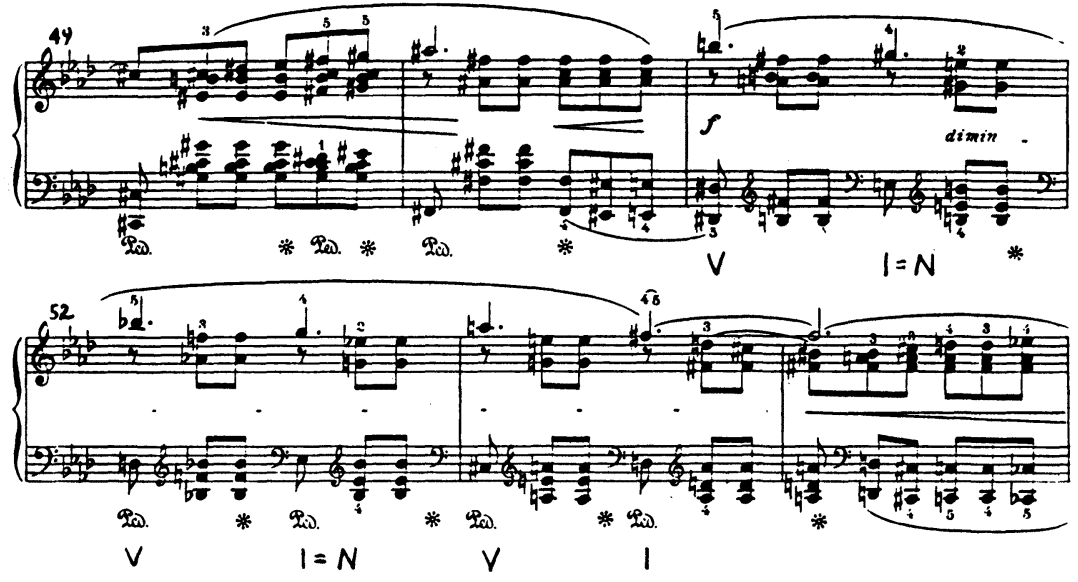

Figure 56. : Chopin : Prélude op. 28, no 17, mes. 51-53. Marche de type no 1. 
L'exemple suivant fait apparaître tantôt II, tantôt N :

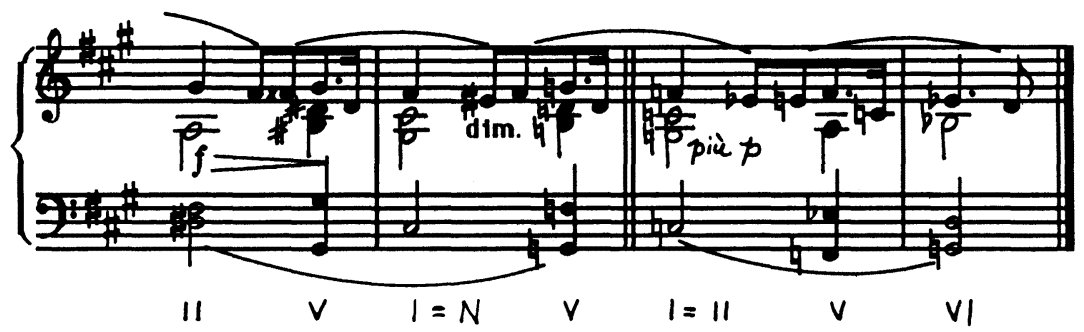

Figure 57. : Wagner : Die Walkure, acte I, scène 1 (fin). Marche de type no 1.

- $\quad \mathrm{V}$ de $\mathrm{V}-\mathrm{V}=\mathrm{V}$ de $\mathrm{V} \ldots$

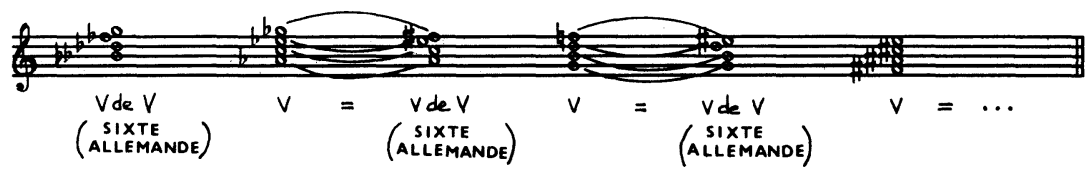

Figure 58. 

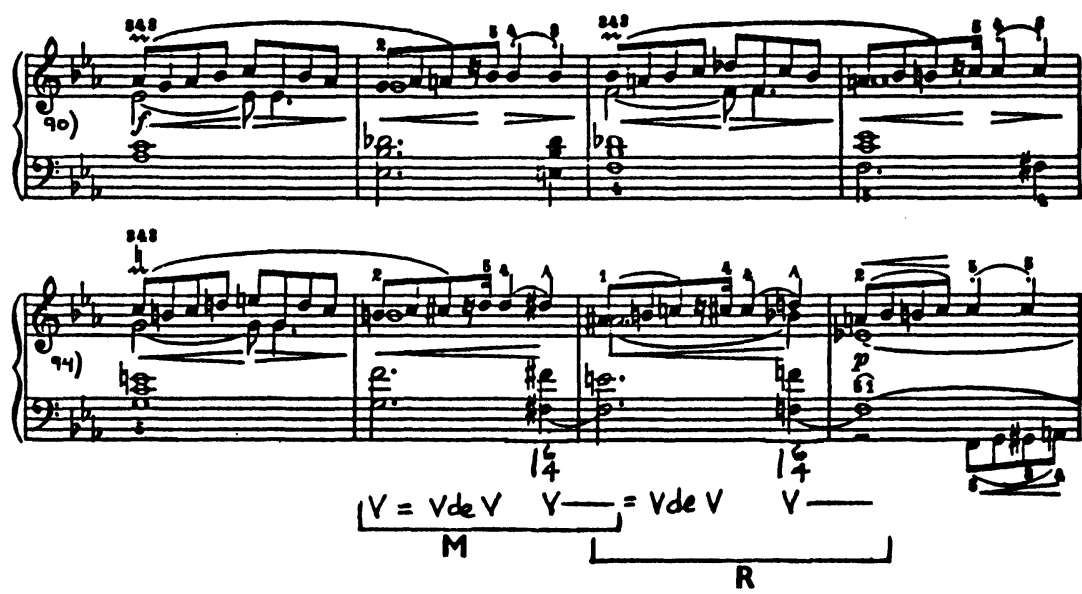

Figure 59. : Chopin : Sonate no 1, I. Marche de type no 1.

\section{La marche de type no 1 sous l'angle non modulant}

Sous l'angle non modulant, la marche de type no 1 peut se présenter :

1. Soit sans fonctions secondaires

c'est-à-dire avec les seuls accords constitutifs (à l'état fondamental ou autrement) auxquels peuvent s'ajouter des accords altérés relevant de l'emploi de la sixte napolitaine ou de l'emploi du mode mixte ;11 quelques formulations en résultant ont déjà été observées.

11 Le mode mixte désigne ce jeu de libre-échange qui survient, à l'intérieur d'un même fragment de discours harmonique, entre les accords de deux modes opposés (majeur et mineur) correspondant à la même tonique. 
2. Soit avec fonctions secondaires ${ }^{12}$

Les plus fréquentes d'entre elles sont, de loin, les dominantes secondaires qui surviennent sous la forme de l'une ou de l'autre des sept structures déjà relevées $: 13$
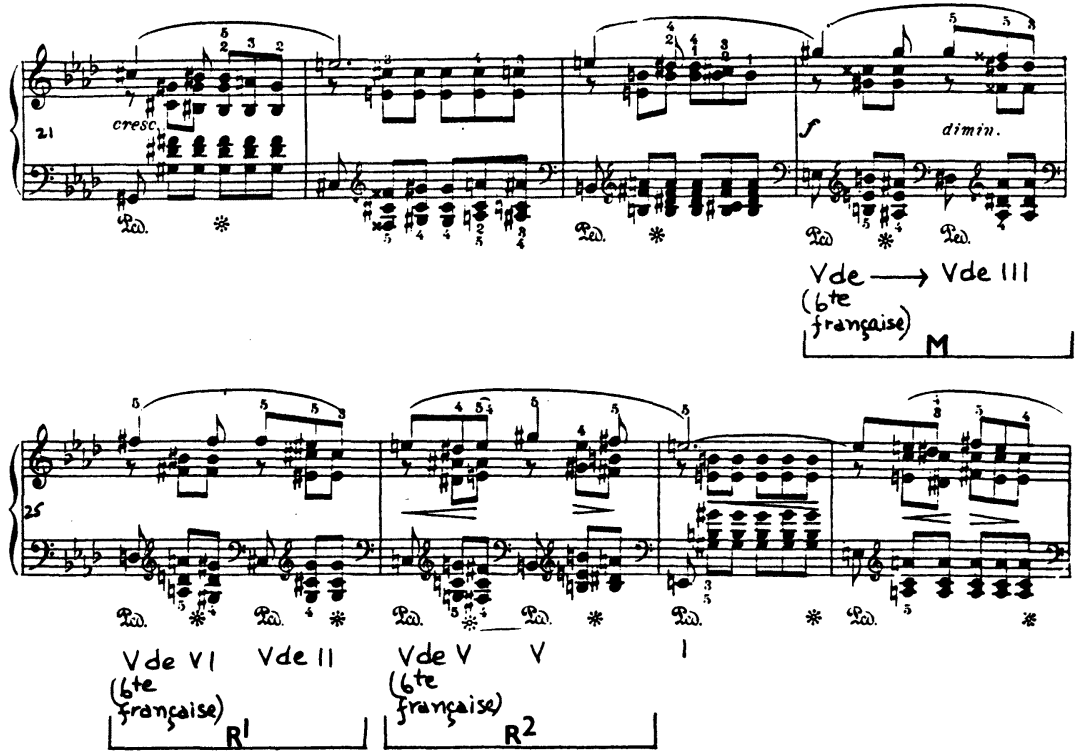

Figure 60. : Chopin : Prélude op. 28, no 17, mes. 24-27. Marche de type no 1.

Mais la polarisation secondaire peut s'étendre et provoquer des fonctions secondaires autres que des dominantes :

12 Les fonctions secondaires sont des fonctions attribuées à des accords qui, dans une perspective non modulante, se rattachent à une tonique secondaire, c'est-à-dire autre que la tonique principale.

13 Cf. exemple 16. 

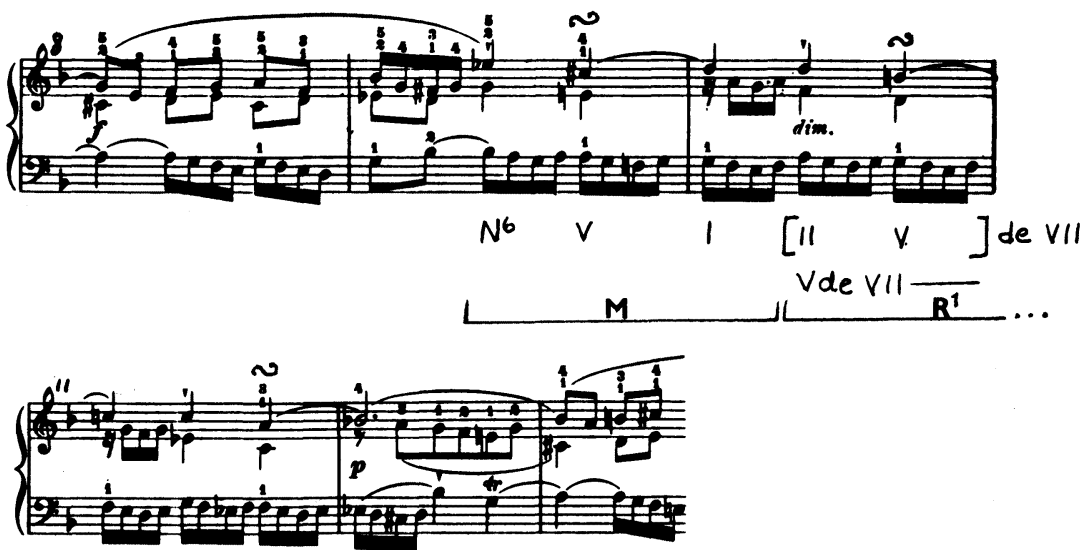

VII $\left[\begin{array}{llllll}11 & V\end{array}\right]$ de VI

$V$ de $V I-\frac{R^{2}}{R^{2}}$

Figure 61. : J. S. Bach : Clavier bien tempéré, vol. I, "Fugue no 6", mes. 9-13. Marche de type no 1. 

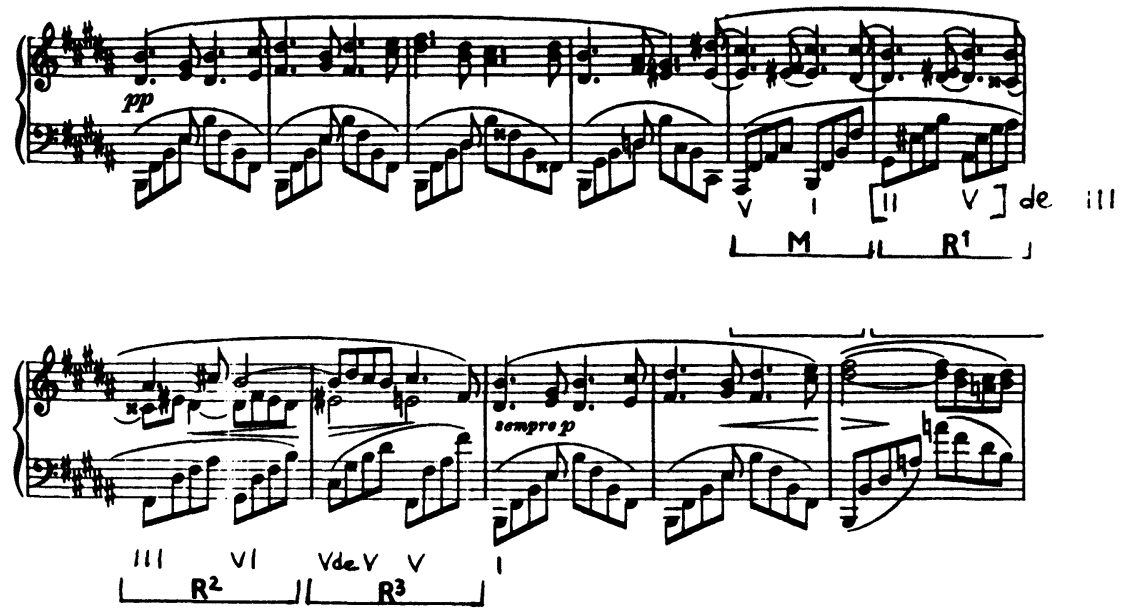

Figure 62. : Brahms : Ballade op. 118, no 3, mes. 46-50. Marche de type no 1. 
La polarisation secondaire peut même, la symétrie s'imposant, se passer de l'énoncé de la tonique secondaire $:^{14}$
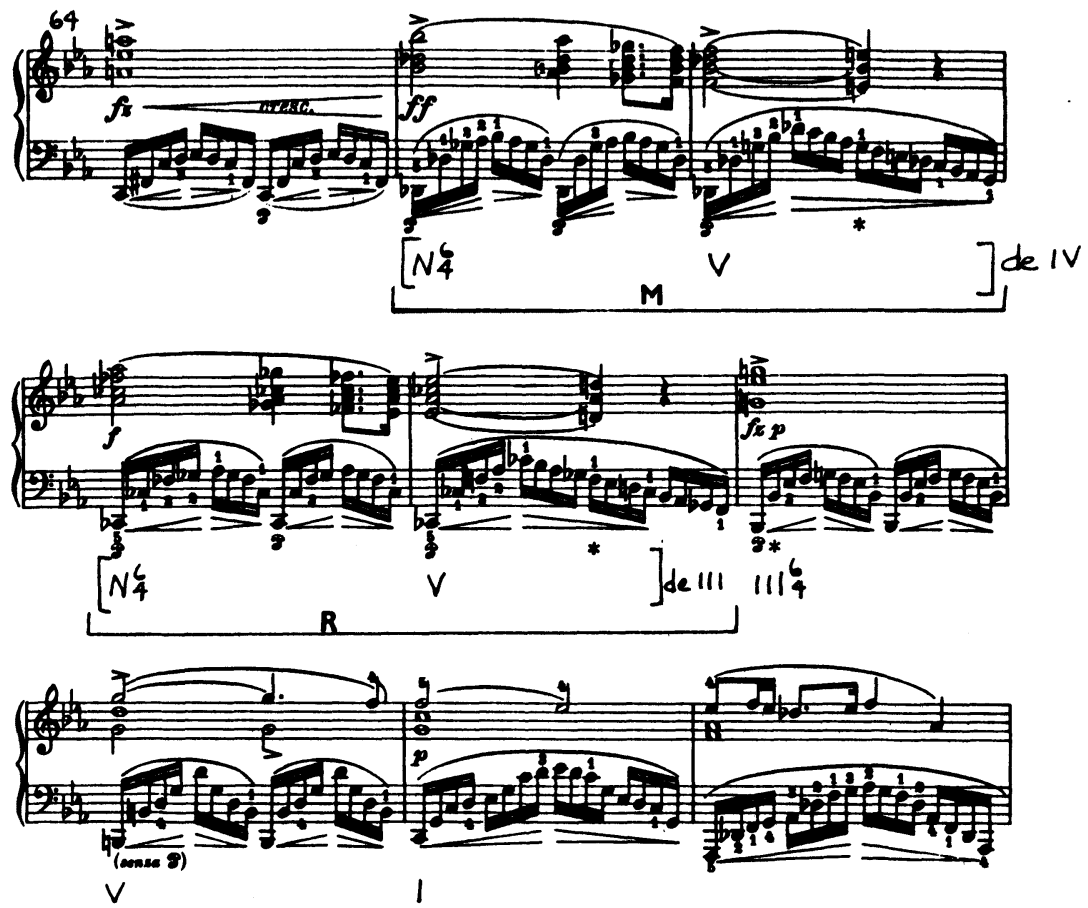

Figure 63. : Chopin : Étude op. 10, no 12, mes. 65-71. Marche de type no 1 .

14 À l'imitation de la polarisation principale, qui peut très bien s'établir sans l'énoncé de la tonique principale. En effet, on peut dire qu'une tonalité est nettement déterminée dès le moment où la fonction de dominante est indentifiée comme telle. Voir, en plus de l'exemple 63, l'exemple 80. 
Enfin, l'angle modulant et l'angle non modulant peuvent se trouver juxtaposés à l'occasion d'une enfilade de différentes structures de la marche de type no 1 :

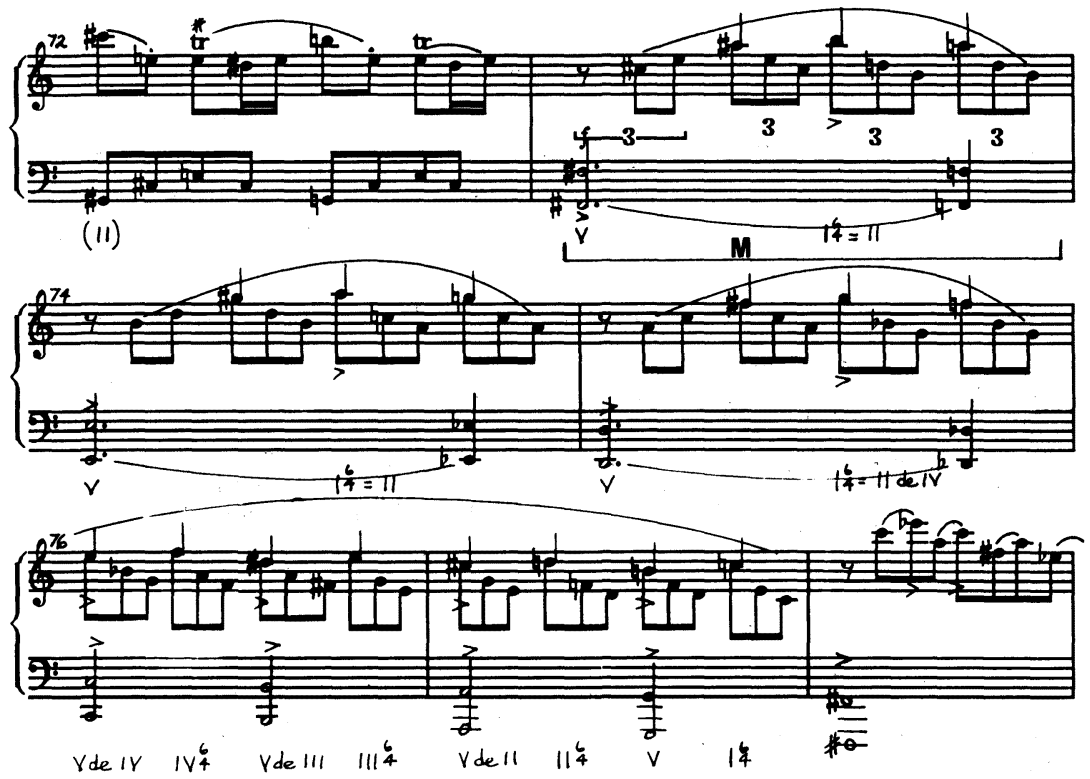

Figure 64. : Mozart : Fantaisie K. 475, mes. 73-77. Marches de type no 1 .

\section{b. Variantes de fond}

Tous les procédés de diversification exposés jusqu'ici n'ont fait que modifier les composantes internes des accords sans altérer d'aucune façon le jeu spécifique des fondamentales. D'autres modes de variation du contenu harmonique vont provoquer des remaniements autrement plus sérieux, en bousculant ce jeu des fondamentales, mais sans toutefois empêcher l'identification de la marche réaménagée. 
C'est, encore une fois, à l'instigation du couple de référence que vont s'introduire ces variantes de fond cherchant à reproduire les substituts les plus fréquents de l'accord de tonique dans le couple dominantetonique. On sait qu'une polarisation principale s'établit souvent à l'aide d'un substitut de la tonique principale. Ainsi en est-il d'une polarisation secondaire, qui va retrouver en ce cas les mêmes substituts, soit ceux que l'on observe au moment de ce que l'on appelle les cadences rompues et les cadences évitées.

Rappelons d'abord que tout accord constitutif d'une tonalité (majeure, mineure ou mixte), sous la forme d'un accord parfait (majeur ou mineur), ainsi que la sixte napolitaine sont susceptibles de remplir le rôle de tonique secondaire (désignée comme $x$ ). Tels qu'ils apparaissent dans les marches, les substituts de $x$ dans le couple $\mathrm{V}$ de $x-x$ secondaire peuvent être ramenés aux deux catégories suivantes :

1. V de $x-\mathrm{V}$ de la fonction suivant $x$ dans le cycle : ler substitut L'accord de tonique secondaire attendu (soit $x$ ) est transformé en dominante secondaire de la fonction suivant $x$ dans le cycle. Cette relation correspond à celle que l'on observe dans la cadence évitée $\mathrm{V}-\mathrm{V}$ de IV, où la fonction de dominante subsiste à condition que soit maintenu le rapport de quinte juste entre les fondamentales des deux accords en cause. Cette dernière observation implique que cette première catégorie de substituts n'entraîne que des variantes de surface qui ont déjà été illustrées dans les exemples 24, 27 et 60 .

Il en va tout autrement de la deuxième catégorie.

2. V de $x$ - VI de $x$ ou IV de $x$ (ou III de $x$ ) : 2e substitut

L'accord de tonique secondaire attendu (soit $x$ ) est remplacé par un accord de sus-dominante ou de sous-dominante de $x$ (plus rarement de médiante de $x$ ). Cette relation correspond à celle que l'on observe dans la cadence rompue (V - VI, V - IV ou V - III). Dans ce cas, au moment de l'interruption de la marche, l'accord agissant comme VI de $x$, ou IV de $x$, (ou III de $x$ ) aura une double fonction, soit une première par rapport à la tonique secondaire et une deuxième par rapport à la tonique principale. C'est à partir de cette deuxième fonction que s'amorcera la formule de clôture. 

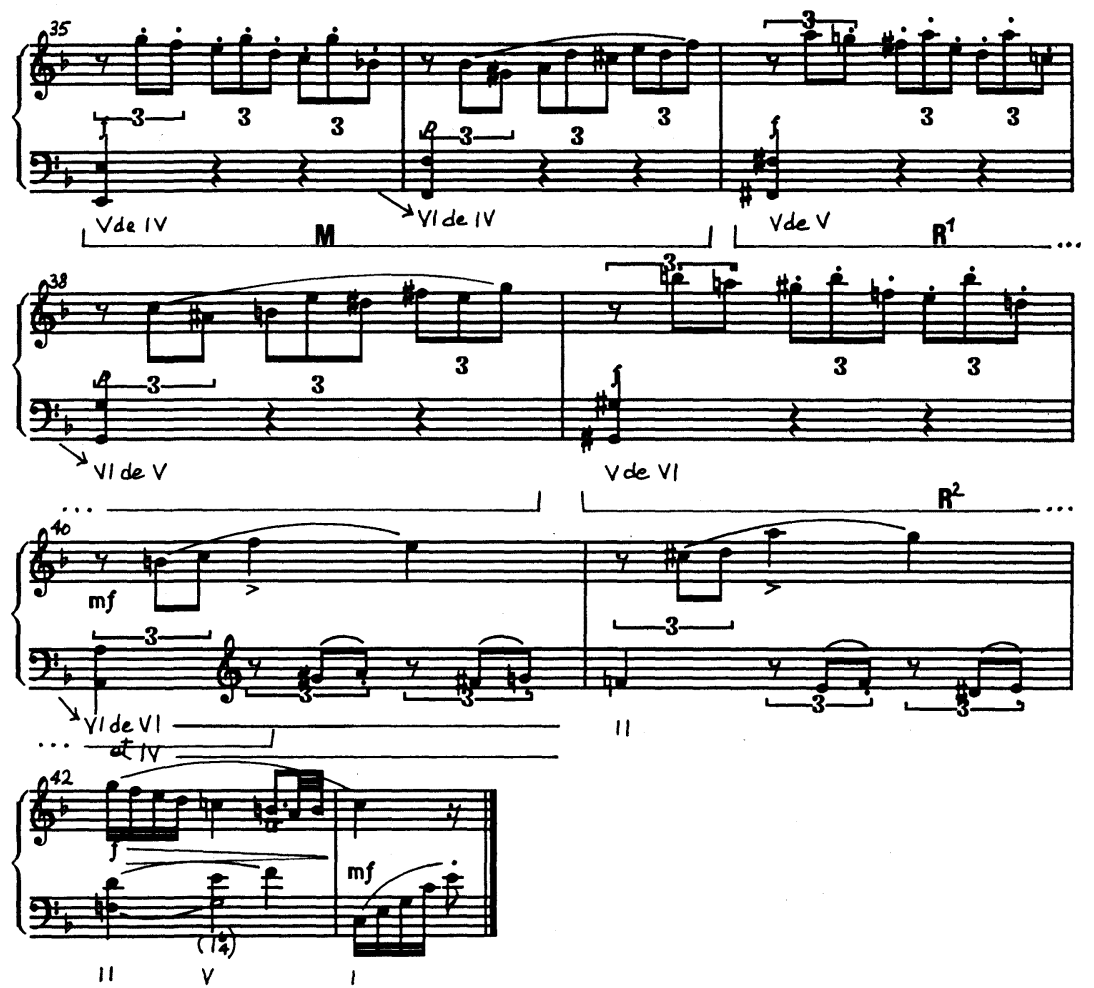

Figure 65. : Mozart : Sonate no 2 (K. 280), I, mes. 35-43. Marche de type no 7. 

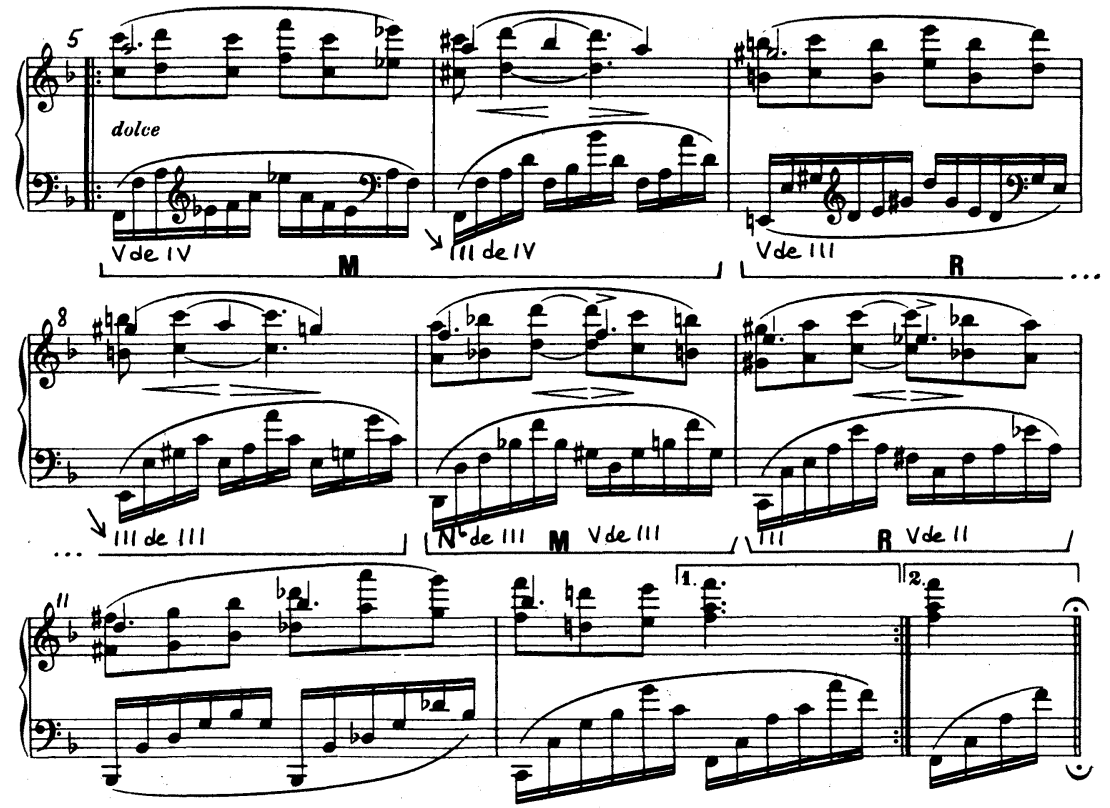

11

$\checkmark$

Figure 66. : Brahms : Variations sur un thème de Paganini, op. 35, var. XII, mes. 5-10. Marches de type no 1.

Dans cet exemple, le dernier III de x (III de III) est suivi d'un autre tronçon de marche no 1 qui démarre en prolongeant la polarisation secondaire au niveau de III. Relevons également dans les exemples 65 et 66 la persistance des motifs mélodiques spécifiques, malgré l'introduction de variantes de fond.

La substitution à la fonction I de la fonction VI dans le couple de référence (substitution de loin la plus fréquente) peut entraîner, en outre, une autre forme d'imitation, indépendante celle-là de toute polarisation secondaire : 


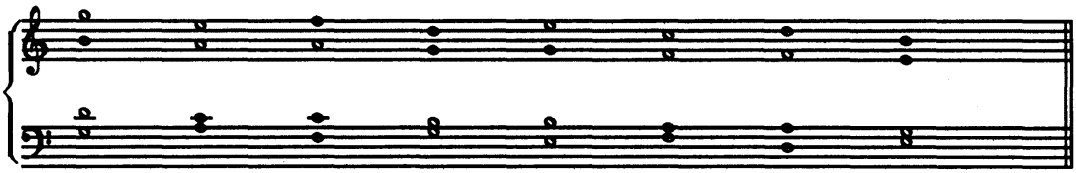

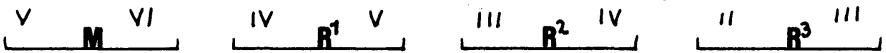

Figure 67.

Là encore, le motif mélodique (aux voix extrêmes) renvoie à la marche de type no 1.
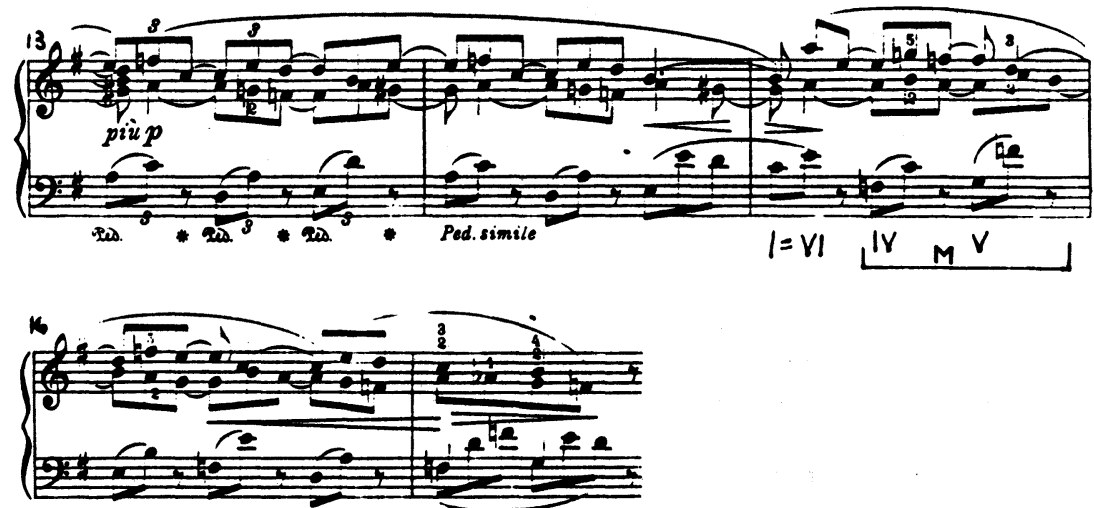

III $R^{1}$ IV III $^{\text {II }}$

Figure 68. : Brahms : Op. 119, no 2, mes. 15-17. Variante du type no 1 .

Enfin, d'autres variantes du contenu des marches sont causées par l'insertion de fonctions à l'intérieur d'un schéma donné correspondant à un type de marche; cette greffe s'inscrit d'ordinaire dans les limites d'un élargissement de la polarisation secondaire : 

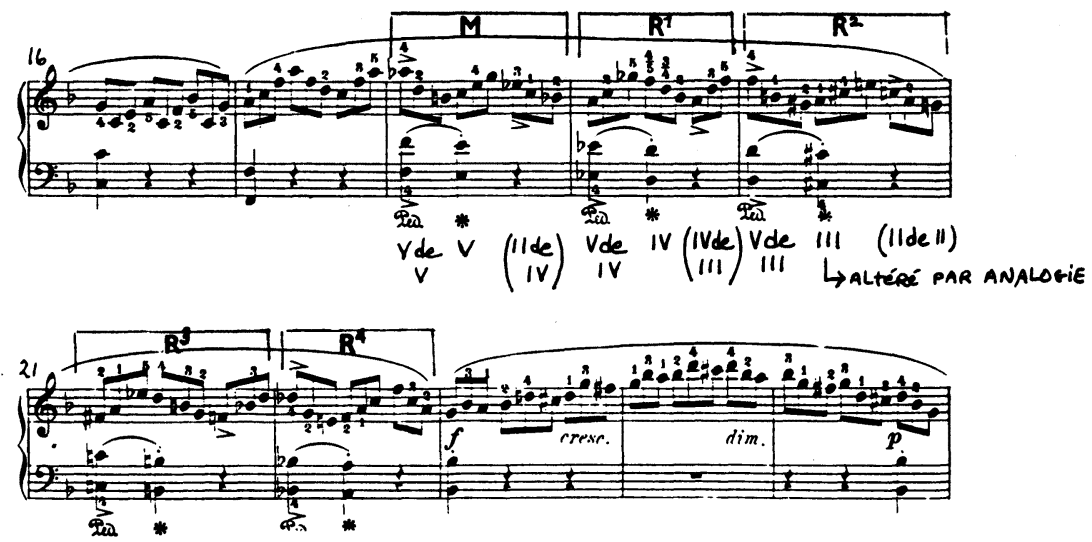

Vde II (IV) $V$ I

II $\rightarrow$ altéré par analogie

Figure 69. : Mozart : Sonate no 2 (K. 280), I, mes. 17-22. Marche de type no 1.

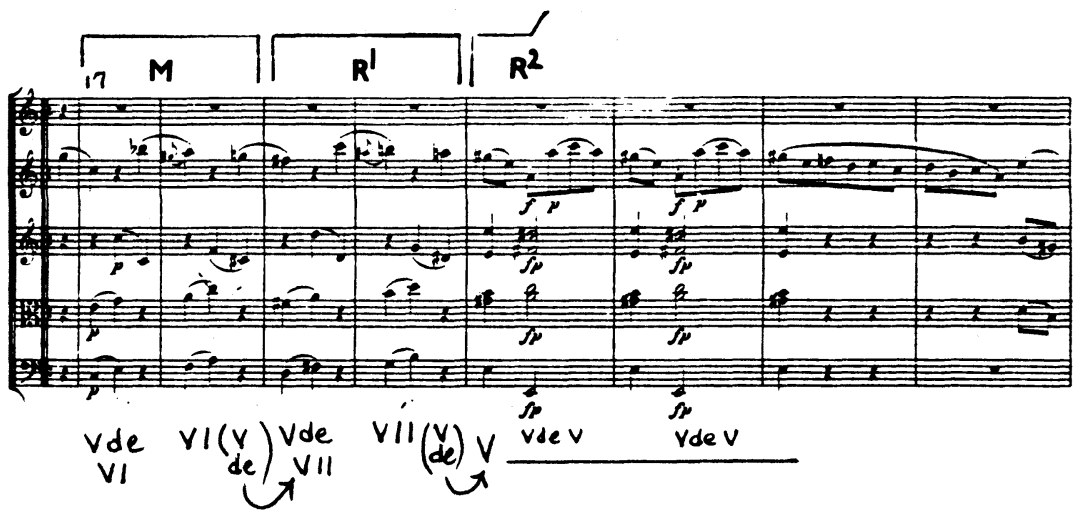

Figure 70. : Mozart : Quintette, K. 580, III, Trio, mes. 17-21. Marche de type no 7. 


\section{LA MARCHE D'HARMONIE COMME LIEU DE SUBVERSION}

Mais, jusqu'où peut aller la variante? Il semble bien que l'utilisation abondante, pour ne pas dire surabondante, de la marche d'harmonie par les compositeurs de musique tonale leur a imposé en quelque sorte de faire montre de plus en plus d'invention, sous peine de banalité. Et c'est ce à quoi ils se sont employés, avec une audace certaine. Certes, il y a là un paradoxe : comment le recours à un stéréotype aussi prégnant dans le discours tonal peut-il se prêter à un renversement de l'ordre? Le paradoxe n'est qu'apparent. C'est justement la force de cette structure, façonnée et reconnue par une longue tradition, qui va leur permettre de faire passer en douce des écarts à toutes sortes de niveaux. Il en est ainsi :

1. Des accords de sixte et quarte à fonction harmonique

C'est dans le cadre d'une marche qu'un accord de sixte et quarte peut revendiquer avec le plus de succès un véritable statut harmonique (plutôt que le statut ornemental qui lui est d'ordinaire alloué) :15

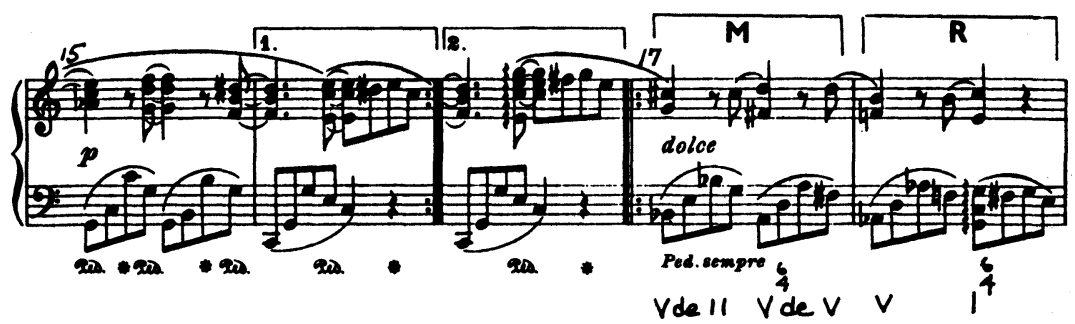

Figure 71. : Brahms : Op. 76, no 7, mes. 17-18. Marche de type no 1 .

2. Des accords altérés par analogie

C'est dans la marche qu'apparaissent des accords altérés sans autre

15 En plus de l'exemple 71, voir à cet égard les exemples 63 et 64 . 
raison que celle de contribuer à la perfection de la symétrie;16 survenant dans un contexte résolument non modulant, ces accords sont inexplicables autrement (sur le plan syntaxique) :17
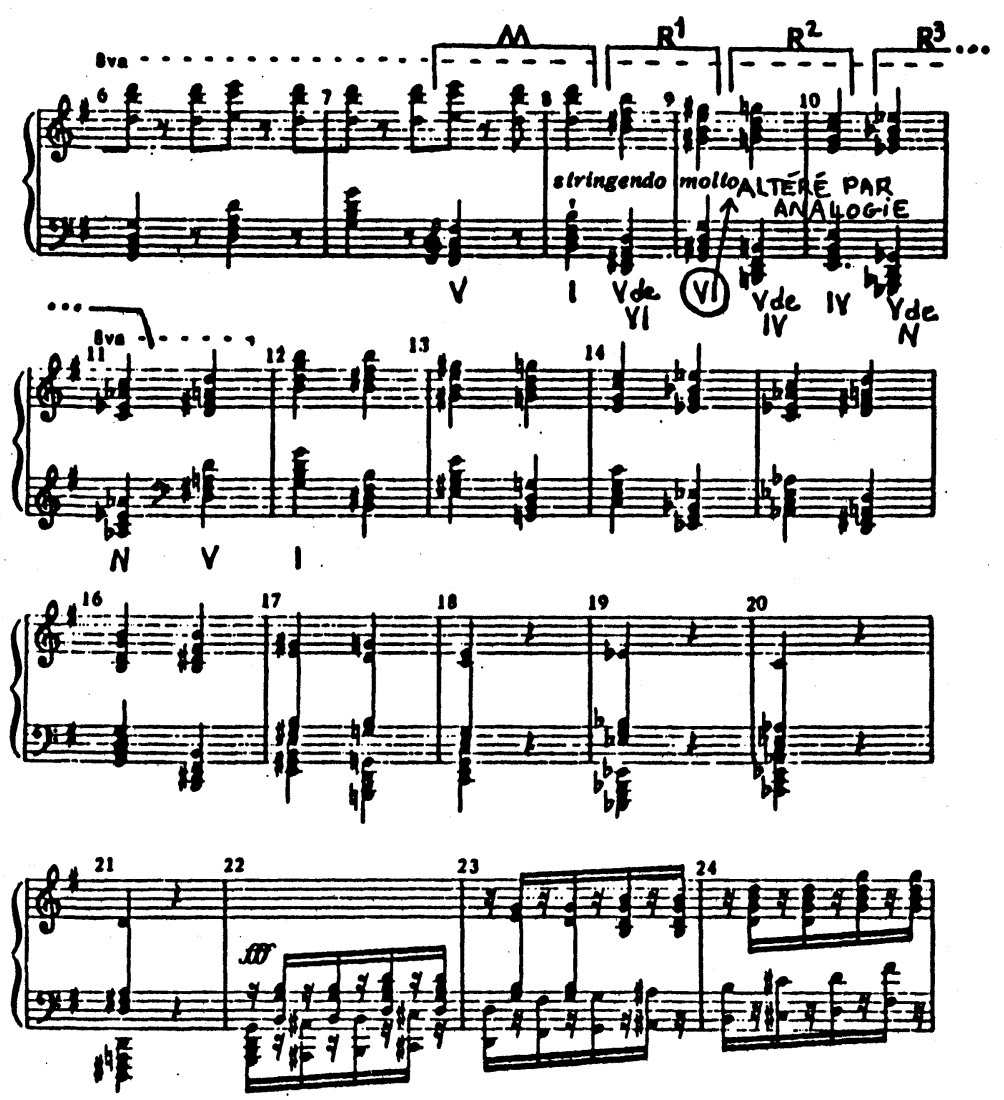

Figure 72. : Liszt : Années de pèlerinage (Venise et Naples), "Tarentella", mes. 8-22. Marche de type no 2.

16 Phénomène relevé par Goldman (1965: 218-19).

17 Voir, en plus des exemples 72 et 73, l'exemple 69. 

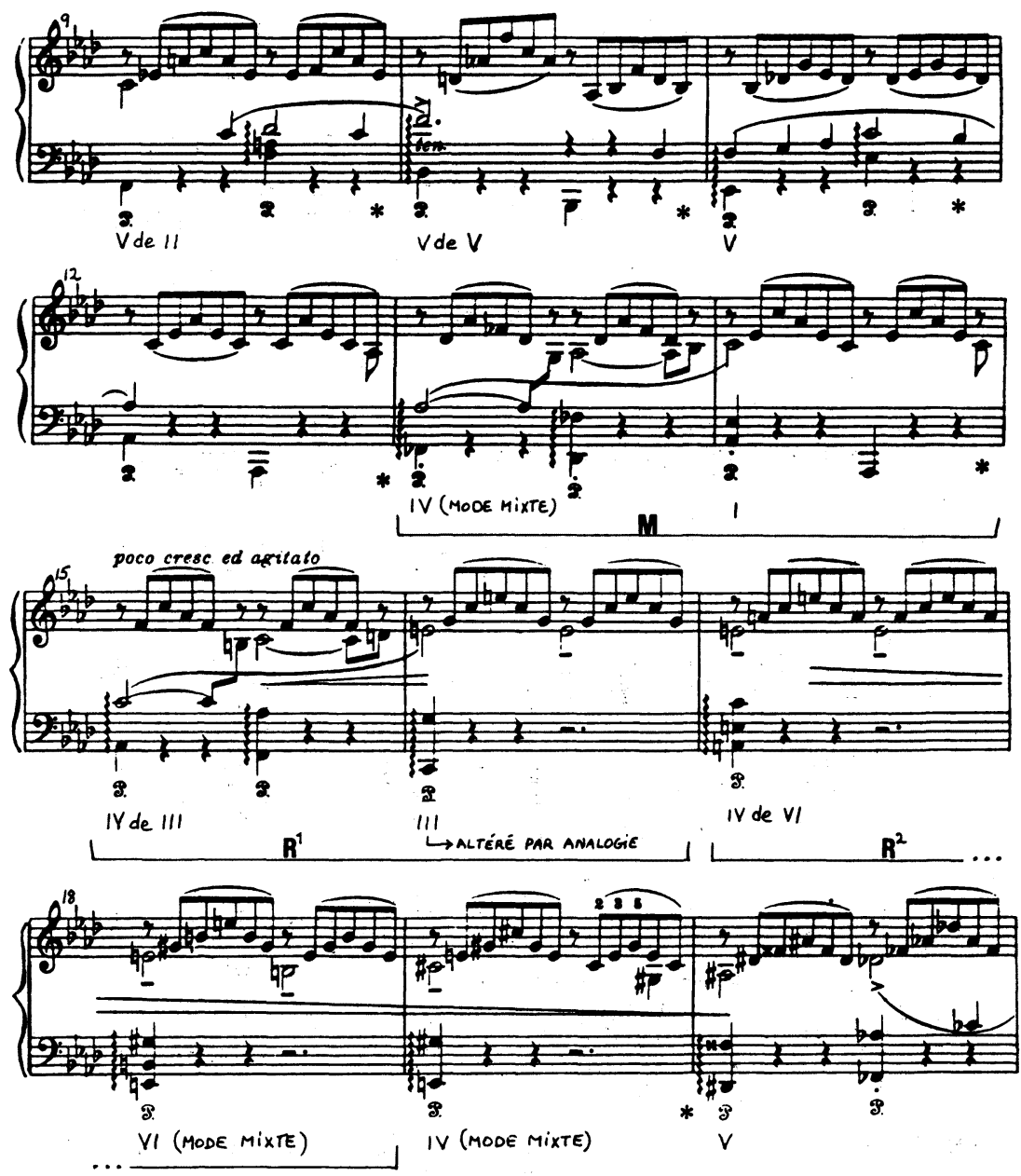

Figure 73. : Liszt : Notturno III, mes. 13-18. Marche de type no 10. 
3. Des couples bizarres

C'est dans la marche qu'on rencontre des couples bizarres ayant toute l'apparence et même acquérant toute la légitimité d'un couple dominante-tonique, à la condition de faire un effort d'interprétation :

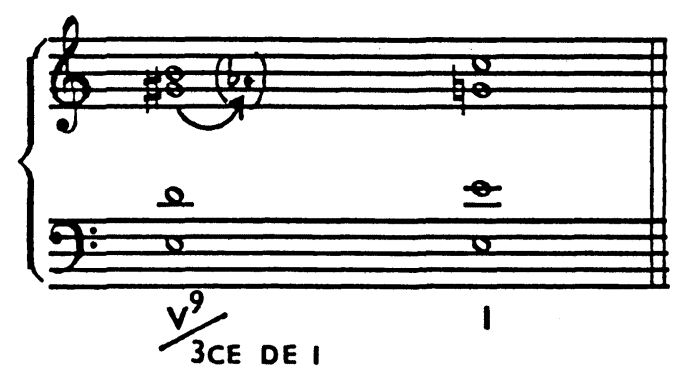

Figure 74.

En ce premier cas, la fonction $\mathrm{V}$ s'explique par le recours à une structure de septième diminuée (si-la bémol-ré) posée sur la tierce (anticipation) de I : 

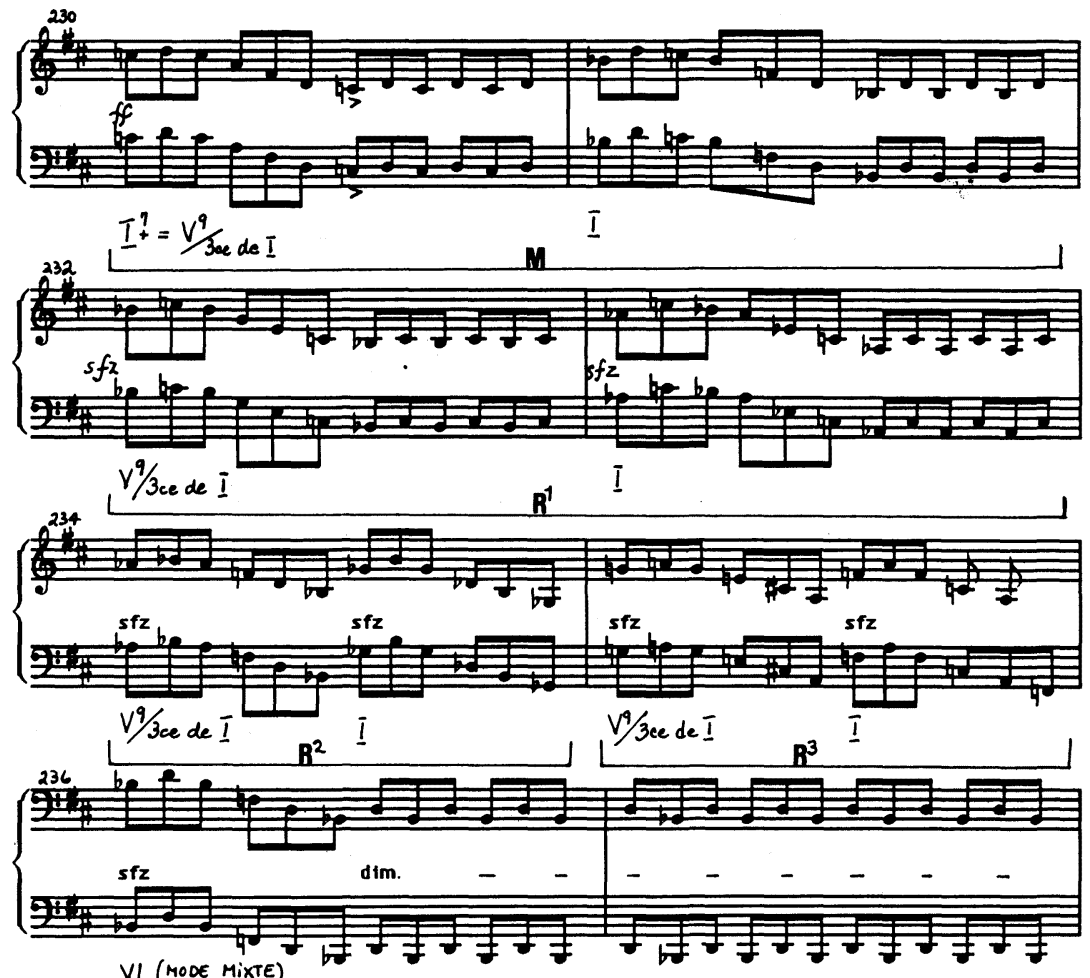

VI (MODE MixTE)

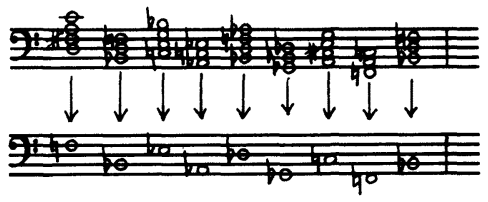

Figure 75. : Schubert : Sonate op. 53, I, mes. 230-235. Marche de type no 1. 
Ce couple pourrait bien sûr relever de l'effet rompu (V - III), mais il arrive que l'étude du contexte incite plutôt à dégager, à son propos, une autre signification. L'examen attentif de tout ce premier mouvement et en particulier des mesures 11-15 et 263-265 permet d'avancer cette interprétation.

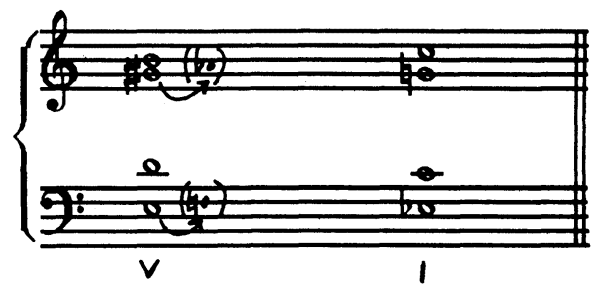

Figure 76. 
En ce deuxième cas, la fonction $\mathrm{V}$ est obtenue par le recours à une structure de septième diminuée dont la septième est altérée, soit abaissée d'un demi-ton :
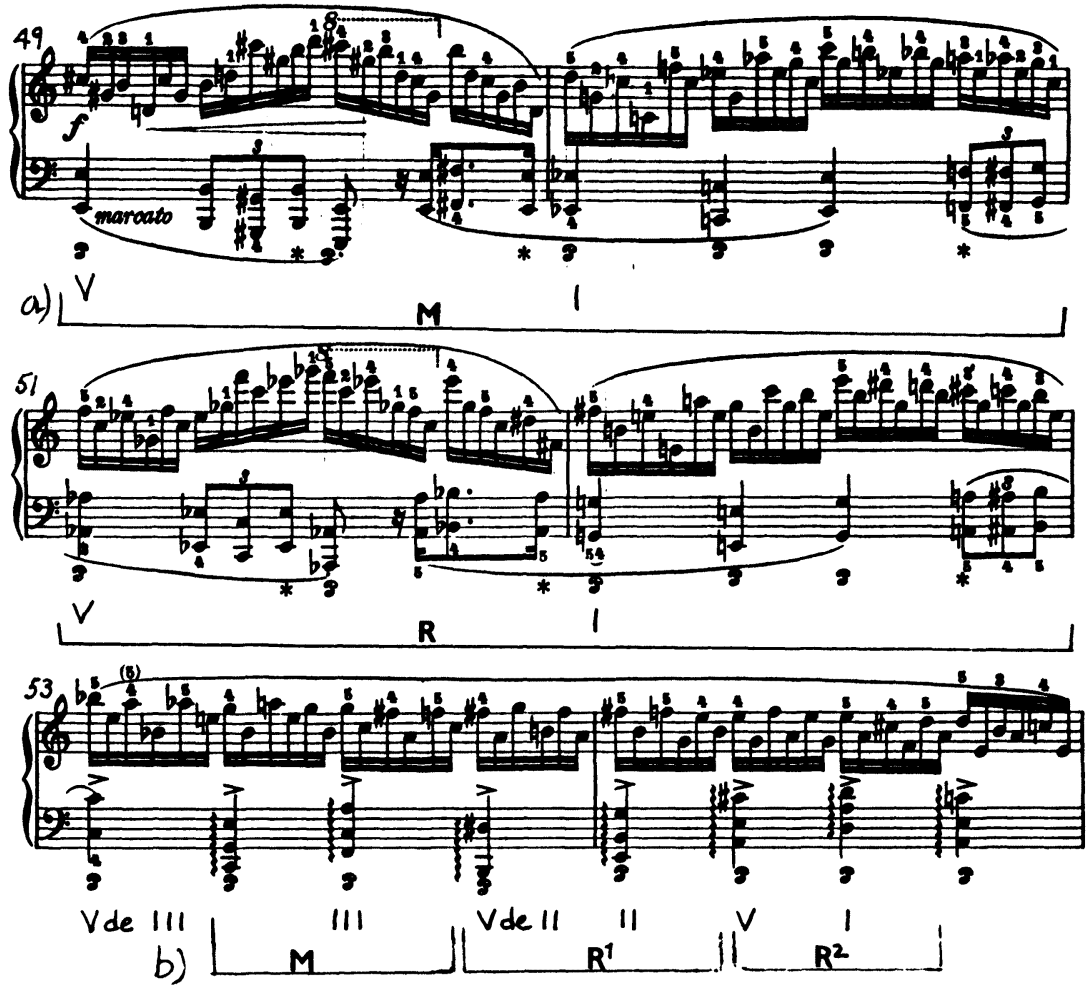

Figure 77. : Chopin : Étude op. 25, no 11, mes. 49-53. Marche de type no 8 (a) et marche de type no 1 (b). 
Le passage précédent renferme le seule geste qui soit en rupture avec le discours harmonique - par ailleurs tout à fait conventionnel - qui est celui des Études de Chopin ${ }^{18}$ (le défi est ailleurs!). Ce geste semble, par-delà le temps, renouer avec le remarquable et singulier début du Moro lasso de Gesualdo :

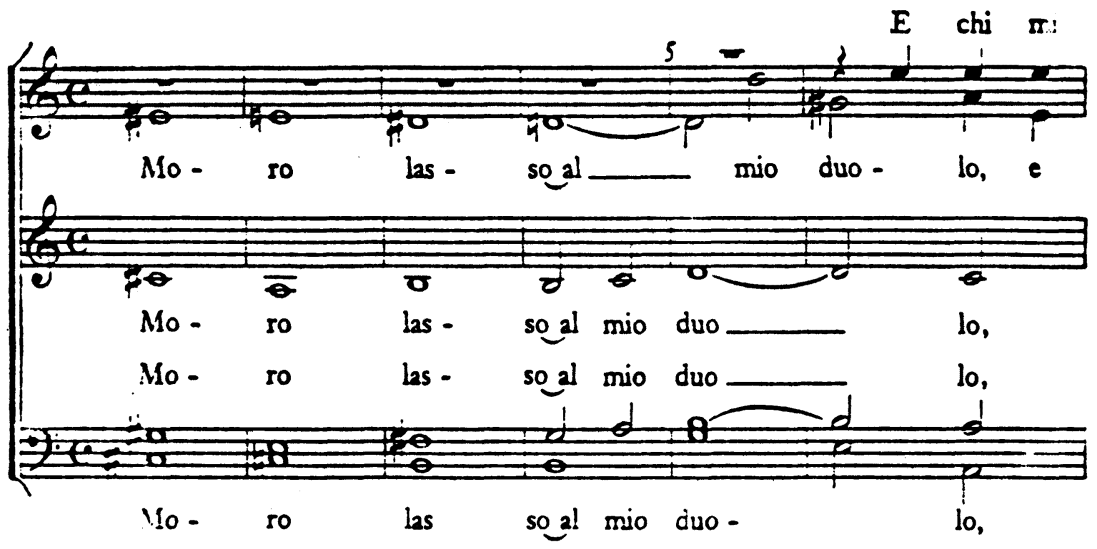

Figure 78. : Gesualdo : Moro lasso, mes. 1-4.

18 Incluant l'opus 10, l'opus 25 et les 3 études posthumes. 
4. Des irrégularités d'entrée dans l'oeuvre

C'est encore la marche d'harmonie qui va permettre, de manière fort insidieuse, de rompre avec la tradition d'entrée dans l'oeuvre :

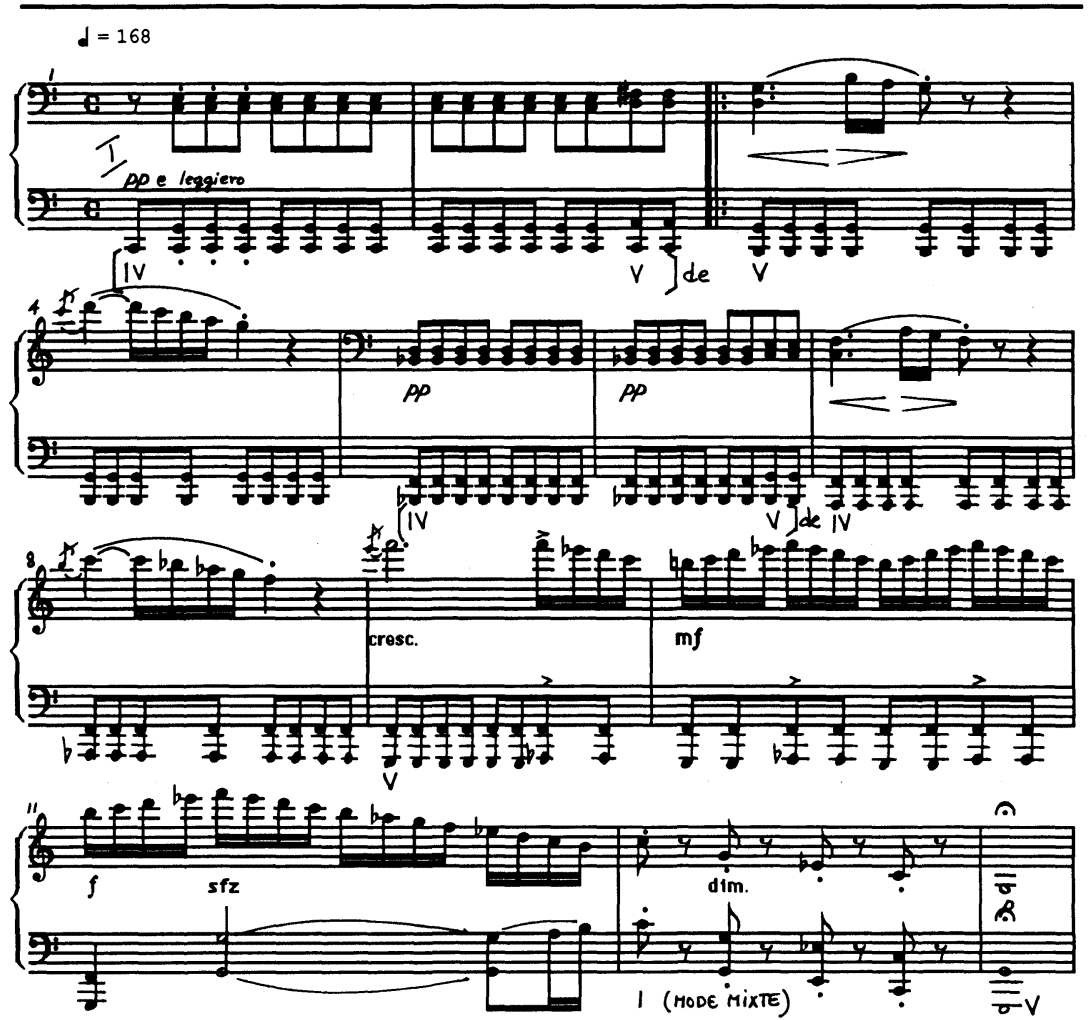

Figure 79. : Beethoven : Sonate op. 53, I, mes. 1-13.

Il semble bien ici que l'oeuvre débute avec l'accord de tonique, par rapport à la tonalité principale. Mais si l'on écoute de près, si l'on regarde de près, dans l'axe de symétrie proposé par la composition, l'on se rend compte que l'on a affaire à un accord de sousdominante de la dominante dans une formule en marche (IV - V - 
I). ${ }^{19}$ Cette perspective met en lumière le rôle joué par l'accord de si bémol majeur à la mesure 5 . Le même phénomène se répète dans l'exemple suivant :

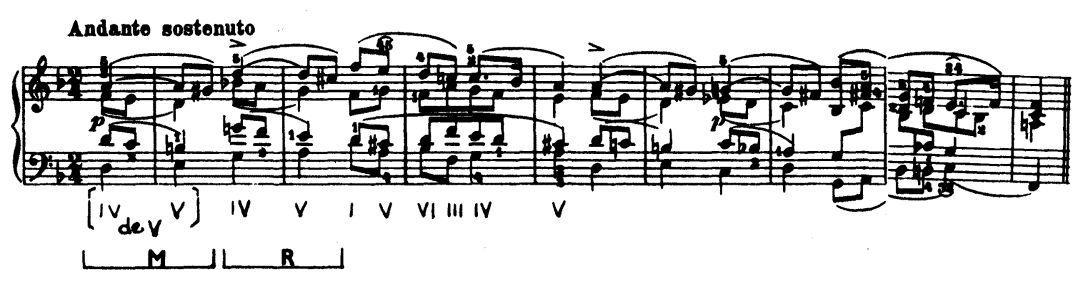

Figure 80. : Mendelssohn : Variations sérieuses, op. 54, mes. $1-8$.

Le même type d'analyse s'impose. Ce premier accord (de ré mineur), correspondant à la tonique du ton principal, va d'abord être entendu comme la sous-dominante de la dominante dans la formule en marche (IV - V).

\section{CONCLUSION}

Bien d'autres faits de même nature ou de nature différente mériteraient d'être relevés à propos de la marche d'harmonie dans la musique tonale, depuis Bach jusqu'à Wagner. Ils participent somme toute de la même attitude à tenter de s'affranchir, à l'intérieur de bornes familières, sécurisantes. C'est la condition du plaisir tonal que de pouvoir s'y retrouver. Et si, au moment de conclure, on s'interroge sur le lieu privilégié de la marche d'harmonie, c'est-à-dire sur la portion du discours musical susceptible de la voir apparaître le plus fréquemment, la réponse est simple. La marche d'harmonie est omniprésente de Bach

19 Il faut ajouter cependant que la fonction de tonique du premier accord n'est pas niée pour autant et se trouve opportunément restaurée à la reprise de l'exposition. 
à Wagner. Elle envahit tout. Du préambule au finale et à la coda. De l'elaboration thématique (partie du thème, ou même thème intégral) à la transition et au développement. Dans les petites formes, dans les grandes. Le compositeur de musique tonale n'y échappe pas. L'auditeur non plus. Source de délectation pour les deux indiscutablement, il nous a semblé qu'elle valait d'être décodée.

\section{REFERENCE}

GOLDMAN, R. F.

1965 : Harmony in Western Music. New York : Norton. 